\title{
GEOMETRIC QUANTIZATION; A CRASH COURSE
}

\author{
EUGENE LERMAN
}

\section{Contents}

1. An outline of the notes 1

2. From Newton's law of motion to geometric mechanics in one hour 2

3. Prequantization 4

4. Polarizations 8

5. Prequantization of differential cocycles 11

Appendix A. Elements of category theory 14

Appendix B. Densities 16

References 20

Early in 2011 Sam Evens acting on behalf of the organizers of the summer school on quantization at Notre Dame asked me to give a short series of lectures on geometric quantization. These lectures were meant to prepare a group of graduate mathematics students for talks at the conference on quantization which were to follow the summer school. I was told to assume that the students had attended an introductory course on manifolds. But I was not to assume any prior knowledge of symplectic geometry. The notes that follow resulted from this request. They are a mostly faithful record of four one-hour lectures (except lecture 4). It should be said that there exist many books on geometric quantization starting with Souriau [14], Sniatycki [12], Simms and Woodhouse [13] , Guillemin and Sternberg [7], Wallach [15] and Woodhouse [16] and continuing with Bates and Weinstein [3] and Ginzburg, Guillemin and Karshon [10]. There are also a number of one hundred page surveys on geometric quantization such as the ones by Ali and Englis [1] and by Echeverria-Enriquez et al. [5]. Clearly I could not have squeezed a semester or more worth of mathematics into four lectures. Since I had to pick and choose, I decided to convey the flavor of the subject by proceeding as follows. In the first lecture I tried to explain how to formulate the Newton's law of motion in terms of symplectic geometry. This naturally require an introduction of the notions of symplectic manifolds, Hamiltonian vector fields and Poisson brackets. In lecture 2 I described prequantization. Lecture 3 dealt with polarizations. I have mostly limited myself to real polarizations. In the original version I tried to explain half-forms. Here I stick with densities. In lecture $4 \mathrm{I}$ came back to prequantization and tried to explain why it is more natural to prequantize a differential cocycle rather than just a two form. In other words prequantization is taken up from a more functorial point of view — differential cohomology and stacks. For reasons of space and time the treatment is not very detailed. The notes also contain two appendices: the first one recalls bits and pieces of category theory; the second discusses densities.

Acknowledgments: I thank the organizers of the summer school at Notre Dame for inviting me to give the talks and providing me with a note taker. I thank the referee for a careful reading of the manuscript and a number of helpful suggestions.

\section{An outline of The notes}

The goal of this mini course is — starting with a classical system (which is modeled as a symplectic manifold together with a function called the Hamiltonian) — to produce a quantum system, that is, a collection of (skew)adjoined operators on a Hilbert space.

Here is a more detailed plan of the lectures (the possibly unfamiliar terms are to be defined later in the course):

- We go from Newton's law of motion to a symplectic formulation of classical mechanics, while cutting quite a few corners along the way.

- Next we have a crash course on symplectic geometry. The two key points are:

- A function $h$ on a symplectic manifold $(M, \omega)$ uniquely defines a vector field $\Xi_{h}$ on the manifold $M$. 
- There is a Poisson bracket, that is, an $\mathbb{R}$-bilinear map

$$
C^{\infty}(M) \times C^{\infty}(M) \rightarrow C^{\infty}(M), \quad(f, g) \mapsto\{f, g\},
$$

which has a number of properties. In particular, the bracket $\{f, g\}$ makes $C^{\infty}(M)$ into a Lie algebra.

- Next we'll discuss prequantization: Given a symplectic manifold $(M, \omega)$ and a corresponding Poisson bracket $\{\cdot, \cdot\}$ we want to find/construct a complex line bundle $\pi: \mathbb{L} \rightarrow M$ with a Hermitian inner product $<\cdot, \cdot\rangle$ and a connection $\nabla$ on $L$ such that:

- the connection $\nabla$ preserves the inner product $<\cdot, \cdot>$

$-\operatorname{curv}(\nabla)=(2 \pi \sqrt{-1}) \omega$

Given such a bundle we get a prequantum Hilbert Space $\mathcal{H}_{0}$, which consists of $L^{2}$ sections of $\mathbb{L} \rightarrow M$. We'll observe:

- Each function $f \in C^{\infty}(M)$ defines an operator $Q_{f}: \mathcal{H}_{0} \rightarrow \mathcal{H}_{0}$,

$$
Q_{f}(s)=(2 \pi \sqrt{-1}) f s+\nabla_{\Xi_{f}} s
$$

where $\Xi_{f}$ is the Hamiltonian vector field generated by the function $f$.

- The map

$$
C^{\infty}(M) \stackrel{Q}{\longrightarrow}\left\{\text { skew Hermitian Operators on } \mathcal{H}_{0}\right\}
$$

given by $f \mapsto Q_{f}$ is a map of Lie algebras.

- There is problem with prequantization: quantum mechanics tells us that the space $\mathcal{H}_{0}$ is too big. Here is an example.

Example 1.1. Consider a particle in $\mathbb{R}^{3}$. The corresponding classical phase space is $M=T^{*} \mathbb{R}^{3}$. The associated line bundle $\mathbb{L}=T^{*} \mathbb{R}^{3} \times \mathbb{C}$ is trivial, and the prequantum Hilbert space is $\mathcal{H}_{0}=L^{2}\left(T^{*} \mathbb{R}^{3}, \mathbb{C}\right)$. Physics tells us that what we should have as our quantum phase space the vector space $L^{2}\left(\mathbb{R}^{3}, \mathbb{C}\right)$.

One then uses polarizations to cut the number of variables in half. The introduction of polarizations leads to a number of technical problems. In other words, this is where our trouble really begins.

In the first appendix to the paper we remind the reader what categories, functors, natural transformations and equivalences of categories are. In the second appendix we discuss densities.

\section{From Newton's law of motion to Geometric mechanics in one hour}

Consider a single particle of mass $m$ moving on a line $\mathbb{R}$ subject to a force $F(q, t)$. Newton's law of motion in this case says: the trajectory $q(t)$ of the particles solves the second order ordinary differential equation (ODE):

$$
m \frac{d^{2} q}{d t^{2}}=F\left(q(t), q^{\prime}(t), t\right)
$$

We now make two simplifying assumptions: (1) the force $F$ only depends on position $q$ and (2) the force $F$ is conservative - that is, $F(q)=-V^{\prime}(q)$ for some $V \in C^{\infty}(M)$. Then (2.1) becomes:

$$
m \frac{d^{2} q}{d t^{2}}=-V^{\prime}(q(t))
$$

The standard way to deal with equation (2.2) is to introduce a new variable $p$ ("momentum") so that $p=m \frac{d q}{d t}$ and convert (2.2) into a system of first order ODEs. That is, if $p=m \frac{d q}{d t}$ then $m \frac{d^{2} q}{d t^{2}}=\frac{d p}{d t}$. Thus every solution of

$$
\left\{\begin{array}{l}
\frac{d q}{d t}=\frac{1}{m} p \\
\frac{d p}{d t}=-V^{\prime}(q)
\end{array}\right.
$$

solves (2.2). On the other hand, a solution of (2.3) is an integral curve of a vector field $\Xi(p, q)$, where

$$
\Xi(p, q)=\frac{1}{m} p \frac{\partial}{\partial q}-V^{\prime}(q) \frac{\partial}{\partial p} .
$$

Note: the energy $H(q, p)=\frac{1}{2 m} p^{2}+V(q)$ is conserved, i.e., it is constant along solutions of (2.3). In fact the function $H$ completely determines the vector field $\Xi$ in the following sense. Consider the two-form $\omega=d p \wedge d q$ on $\mathbb{R}^{2}$. It is easy to see that

$$
\iota(\Xi) \omega=-d H
$$


So $\mathrm{H}$ determines $\Xi$. Notice $\omega=d p \wedge d q$ is nondegenerate, so for any 1 -form $\alpha$ the equation $\omega(X, \cdot)=\alpha(\cdot)$ has a unique solution. To summarize: Newton's equations and $m \frac{d^{2} q}{d t}=-V^{\prime}(q)$ are equivalent to integrating the vector field $\Xi_{H}$ defined by $\iota\left(\Xi_{H}\right) \omega=-d H$. We now generalize this observation.

Definition 2.1. A symplectic form $\omega$ on a manifold $M$ is a closed nondegenerate 2 -form. The pair $(M, \omega)$ is called a symplectic manifold.

Remark 2.2. $d \omega=0$ will give us an important property of the Poisson bracket: the Jacobi identity.

\section{Standard examples of symplectic manifolds.}

Example 2.3. $\left(\mathbb{R}^{2}, d p \wedge d q\right)$

Example 2.4. $(\Sigma, \omega)$ where $\Sigma$ is an orientable surface and $\omega$ is an area form (nowhere zero form) on $\Sigma$. Note that since the surface $\Sigma$ is two dimensional, $d \omega$ is automatically 0 .

Example 2.5. Let $Q$ be any manifold and set $M=T^{*} Q$. If $\alpha$ denotes the tautological 1 -form then $\omega=d \alpha$ is a symplectic form on $M$. Here are some details. "Recall" that there are two ways of defining the tautological 1-form (also called the Liouville form) $\alpha$.

(1) In local coordinates $\alpha$ is defined as follows. If $\left(q_{1}, \ldots q_{n}\right)$ is a coordinate chart on $Q$ and $\left(q_{1}, \ldots, q_{n}, p_{1}, \ldots, p_{n}\right)$ the corresponding coordinates on the cotangent bundle $T^{*} M$, then

$$
\alpha=\sum p_{i} d q_{i} .
$$

It is not hard to check that $d \alpha=\omega=\sum d p_{i} \wedge d q_{i}$ is non-degenerate. It is closed by construction, hence it's symplectic. It is not obvious that $\alpha$ (and hence $\omega$ ) are globally defined forms.

(2) Alternatively, we have the projection $\pi: T^{*} Q \rightarrow Q$ and $d \pi: T_{(q, p)}\left(T^{*} Q\right) \rightarrow T_{q} Q$. So given $q \in Q, p \in T_{q}^{*} Q$ and $v \in T_{(q, p)}\left(T^{*} Q\right)$ define

$$
\alpha_{(q, p)}(v)=p(d \pi(v)) .
$$

It is a standard exercise to check that the two constructions agree. In the first construction of $\alpha$ it is clear that $\alpha$ is smooth and $d \alpha$ is nondegenerate. In the second construction it is clear that $\alpha$ is globally defined.

Definition 2.6. The Hamiltonian vector field $\Xi_{f}$ of a function $f$ on a symplectic manifold $(M, \omega)$ is the unique vector field defined by $\omega\left(\Xi_{f}, \cdot\right)=-d f$.

Warning: the opposite sign convention is also frequently used in literature: $\omega\left(\Xi_{f}, \cdot\right)=d f$.

Remark 2.7. The function $f$ is always constant along the integral curves of its Hamiltonian vector field $\Xi_{f}$.

Proof. Let $\gamma$ be an integral curve of the vector field $\Xi_{f}$. Then

$$
\frac{d}{d t} f(\gamma(t))=\Xi_{f}(f)=d f\left(\Xi_{f}\right)=-\omega\left(\Xi_{f}, \cdot\right)\left(\Xi_{f}\right)=-\omega\left(\Xi_{f}, \Xi_{f}\right) .
$$

Since $\omega$ is skew-symmetric, $\omega\left(\Xi_{f}, \Xi_{f}\right)=0$. Hence $\frac{d}{d t} f(\gamma(t))=0$, i.e., $f(\gamma(t))$ is a constant function of $t$, which is what we wanted to prove.

\section{Poisson Bracket.}

Definition 2.8. The Poisson bracket $\{\cdot, \cdot\}$ on a symplectic manifold $(M, \omega)$ is a map

$$
\{\cdot, \cdot\}: C^{\infty}(M) \times C^{\infty}(M) \rightarrow C^{\infty}(M)
$$

defined by

$$
\{f, g\}=\Xi_{f}(g) .
$$

Remark 2.9. The Poisson bracket has a number of useful properties which we list below. Proofs may be found in any symplectic geometry book.

(1) For any three function $f, g, h \in C^{\infty}(M)$ we have

$$
\{f, g \cdot h\}=\Xi_{f}(g \cdot h)=\Xi_{f}(g) \cdot h+g \cdot \Xi_{f}(h)=\{f, g\} \cdot h+g \cdot\{f, h\} .
$$

(2) For any pair of functions $f, g$ we have $\{f, g\}=\Xi_{f}(g)=d g\left(\Xi_{f}\right)=-\omega\left(\Xi_{g}, \Xi_{f}\right)=\omega\left(\Xi_{f}, \Xi_{g}\right)=-\{g, f\}$. In particular $\{f, f\}=0$. 
(3) One can show that the equation $d \omega=0$ implies that

$$
\{f,\{g, h\}\}=\{\{f, g\}, h\}+\{g,\{f, g\}\},
$$

which is the Jacobi identity. In other words the pair $\left(C^{\infty}(M),\{\cdot, \cdot\}\right)$ is a Lie algebra.

(4) It is not hard to show that $\{f, g\}=0$ if and only if $g$ is constant along integral curves of $f$. Indeed, let $\gamma$ be an integral curve of the vector field $\Xi_{f}$. Then

$$
\frac{d}{d t} g(\gamma(t))=\Xi_{f}(g)(\gamma(t))=\{f, g\}(\gamma(t))
$$

Therefore if the function $g$ is constant along $\gamma$ then the bracket $\{f, g\}$ is zero along $\gamma$. The converse is true as well. This generalizes the fact that a function $f$ is constant along the integral curves of its Hamiltonian vector field $\Xi_{f}$.

(5) One can show that $\iota\left(\left[\Xi_{f}, \Xi_{g}\right]\right) \omega=-d\{f, g\}$. Hence if the Poisson bracket $\{f, g\}$ of two functions $f, g$ is 0 then flows of their Hamiltonian vector fields commute. Here is a better interpretation of the same fact: The map

$C^{\infty}(M) \rightarrow$ vector fields on $M, \quad f \mapsto \Xi_{f}$

is a map of Lie algebras: $\Xi_{\{f, g\}}=\left[\Xi_{f}, \Xi_{g}\right]$.

Remark 2.10. It is not hard to show that is $(M, \omega)$ is a symplectic manifold, then its dimension is necessarily even. This only involves linear algebra.

Say $\operatorname{dim} M=2 n$. Then one can show further that the $2 n$-form $\omega^{n}:=\overbrace{\omega \wedge \cdots \wedge \omega}^{n}$ (n-fold wedge product) is nowhere zero, hence defines an orientation of $M$. In particular, this allows us to integrate any compactly supported function $f \in C^{\infty}(M)$ over $M$ by integrating the form $f \omega^{n}$. The space $L^{2}(M, \omega)$ is then defined as the completion of the space $C_{c}^{\infty}(M, \mathbb{C})$ of compactly supported functions with respect to the $L^{2}$ norm

$$
\|f\|:=\left(\int_{M}|f|^{2} \omega^{n}\right)^{1 / 2}
$$

It is a Hilbert space with the Hermitian inner product

$$
\langle\langle f, g\rangle\rangle:=\int_{M} \bar{f} g \omega^{n}
$$

(in the convention I prefer, the Hermitian inner products are complex-linear in the second variable).

We end the section with an easy lemma that we will need later.

Lemma 2.11. Let $(M, \omega)$ be a symplectic manifold and $f: M \rightarrow \mathbb{R}$ a smooth function. Then the Lie derivative $\mathcal{L}_{\Xi_{f}} \omega$ of the symplectic form with respect to the Hamiltonian vector field of the function $f$ is zero:

$$
\mathcal{L}_{\Xi_{f}} \omega=0
$$

Proof. This is an application of Cartan's formula: for a differential form $\sigma$ its Lie derivative $\mathcal{L}_{X} \sigma$ with respect to a vector field $X$ is given by $\mathcal{L}_{X} \sigma=\iota(X) d \sigma+d \iota(X) \sigma$, where, as above, $\iota(X) \sigma$ denotes the contraction of $X$ and $\sigma$, etc. Thus

$$
\mathcal{L}_{\Xi_{f}} \omega=\iota\left(\Xi_{f}\right) d \omega+d \iota\left(\Xi_{f}\right) \omega .
$$

The first summand above is 0 since $d \omega=0$. By definition of $\Xi_{f}, \iota\left(\Xi_{f}\right) \omega=-d f$. Since $d(d f)=0$, the second summand is zero as well.

\section{PREQUANTIZATion}

The goal of this section is to describe geometric prequantization. This is a procedure for turning a classical mechanical system mathematically formalized as a symplectic manifold $(M, \omega)$ together with its Poisson algebra of smooth functions $C^{\infty}(M)$ ("classical observables") into a quantum system formalized as a Hilbert space $\mathcal{H}_{0}$ together with the Lie algebra of (densely defined) skew-Hermitian operators $\left\{Q_{f} \mid f \in C^{\infty}(M)\right\}$. Moreover the map

$$
Q: C^{\infty}(M) \rightarrow \operatorname{End}\left(\mathcal{H}_{0}\right), \quad f \mapsto Q_{f},
$$

should (and would) be a map of Lie algebras:

$$
Q_{\{f, g\}}=\left[Q_{f}, Q_{g}\right]
$$

for all functions $f, g \in C^{\infty}(M)$. We start by recalling some notation.

Notation 3.1. We denote the space of sections of a vector bundle $E \rightarrow M$ over a manifold $M$ by $\Gamma(E)$. Thus the space of vector fields on a manifold $M$ is denoted by $\Gamma(T M)$. 
3.1. Connections. We start by recalling a number of standard definitions and facts. By a fact I mean a theorem the proof of which will take us too far afield. Such proofs may be found in any number of textbooks.

Definition 3.2. A connection $\nabla$ on a (complex) vector bundle $E \stackrel{\pi}{\rightarrow} M$ is a $\mathbb{C}$-bilinear map

$$
\Gamma(T M) \times \Gamma(E) \rightarrow \Gamma(E), \quad(X, s) \mapsto \nabla_{X} s
$$

such that

(1) $\nabla_{f X} s=f \nabla_{X} s$ for all functions $f \in C^{\infty}(M, \mathbb{C})$ and all vector fields $X \in \Gamma(T M)$ (i.e., $\nabla$ is $C^{\infty}(M, \mathbb{C})$ linear in the first variable) and

(2) $\nabla_{X}(f s)=X(f) s+f \nabla_{X} s$ for all functions $f \in C^{\infty}(M, \mathbb{C})$ and all vector fields $X \in \Gamma(T M)$ (i.e., $\nabla$ is a derivation in second slot).

If the vector bundle $E$ carries a fiber-wise Hermitian inner product $\langle\cdot, \cdot\rangle$ we can talk about the connections respecting this structure. More precisely

Definition 3.3. A connection $\nabla$ on a vector bundle $E \stackrel{\pi}{\rightarrow} M$ with a fiber-wise inner product $\langle\cdot, \cdot\rangle$ is Hermitian if

$$
X\left(\left\langle s, s^{\prime}\right\rangle\right)=\left\langle\nabla_{X} s, s^{\prime}\right\rangle+\left\langle s, \nabla_{X} s^{\prime}\right\rangle
$$

for all vector fields $X$ on $M$ and all section $s, s^{\prime} \in \Gamma(E)$.

Next recall that given any complex vector bundle $E \stackrel{\pi}{\rightarrow} M$ we can consider the bundle $\operatorname{End}(E) \rightarrow M$ of endomorphisms with a fiber $\operatorname{End}(E)_{x}$ at a point $x \in M$ given by

$$
\operatorname{End}(E)_{x}=\left\{A: E_{x} \rightarrow E_{x} \mid A \text { is } \mathbb{C} \text { linear }\right\} .
$$

We also have the subbundle of skew Hermitian maps $\operatorname{End}(E,\langle\cdot, \cdot\rangle) \subset \operatorname{End}(E)$ with typical fiber

$$
\operatorname{End}(E,\langle\cdot, \cdot\rangle)_{x}=\left\{A: E_{x} \rightarrow E_{x} \mid A \text { is } \mathbb{C} \text { linear and }\langle A v, w\rangle+\langle w, A v\rangle=0 \quad \forall v, w \in E_{x}\right\} .
$$

We have the following Fact:

Fact 3.4. The space of Hermitian connections on a vector bundle $(E,\langle\cdot, \cdot\rangle)$ is non empty. In fact it is an infinite dimensional affine space: the difference of two connections is a $\operatorname{End}(E,\langle\cdot, \cdot\rangle)$ valued 1 -form.

Definition 3.5. Let $\nabla: \Gamma(T M) \times \Gamma(E) \rightarrow \Gamma(E)$ be a connection on a vector bundle $E \rightarrow M$. The curvature $R^{\nabla}$ of the connection is a section of $\Lambda^{2}\left(T^{*} M\right) \otimes \operatorname{End}(E)$ (i.e., an $\operatorname{End}(E)$ valued 2-form). It is defined by

$$
R^{\nabla}(X, Y) s=\nabla_{X}\left(\nabla_{Y} s\right)-\nabla_{Y}\left(\nabla_{X} s\right)-\nabla_{[X, Y]} s
$$

for all vector fields $X$ and $Y$ and all sections $s \in \Gamma(E)$.

If $\nabla$ is a Hermitian connection then its curvature $R^{\nabla}$ is a 2 -form with values in $\operatorname{End}(E,\langle\cdot, \cdot\rangle)$. Furthermore if $E \stackrel{\pi}{\rightarrow} M$ is a complex line bundle then

hence

$$
\operatorname{End}(E,\langle\cdot, \cdot\rangle) \simeq M \times \sqrt{-1} \mathbb{R}
$$

$$
\frac{1}{\sqrt{-1}} R^{\nabla} \in \Omega^{2}(M, \mathbb{R})
$$

That is $\frac{1}{\sqrt{-1}} R^{\nabla}$ is an ordinary real valued 2 -form.

Fix a manifold $M$ and consider the collection $\mathcal{D}(M)$ of all triples $(L,\langle\cdot, \cdot\rangle, \nabla)$, where $L \rightarrow M$ is a complex line bundle, $\langle\cdot, \cdot\rangle$ is a Hermitian inner product on $L$ and $\nabla$ is a Hermitian connection on $(L,\langle\cdot, \cdot\rangle)$. Then curvature defines a map

$$
\mathcal{D}(M) \rightarrow \Omega^{2}(M), \quad(L,\langle\cdot, \cdot\rangle, \nabla) \mapsto \frac{1}{\sqrt{-1}} R^{\nabla}
$$

from the collection $\mathcal{D}(M)$ to the set of (real-valued) 2-forms $\Omega^{2}(M)$.

To define geometric prequantization one needs to invert this map. That is, given a symplectic manifold $(M, \omega)$ one would like to find a Hermitian line bundle with a Hermitian connection $\nabla$ so that

$$
\frac{1}{\sqrt{-1}} R^{\nabla}=\omega \text {. }
$$

However there are two problems: (1) the map (3.1) is not 1-1 and (2) not all symplectic forms are in the image of the map. The first problem has to do with the fact that taking curvature of a connection is very much like taking the exterior derivative of a 1-form. So recovering connection from its curvature is also like recovering a 1-form from its exterior derivative. 
The second problem is topological. It has to do with the fact that (isomorphism classes of) complex line bundles are parametrized by degree 2 integral cohomology classes, that is, elements of $H^{2}(M, \mathbb{Z})$. Moreover the cohomology class $c_{1}(M)$ of a line bundle $E \rightarrow M$ and the de Rham class $\left[\frac{1}{2 \pi \sqrt{-1}} R^{\nabla}\right]$ defined by the curvature $R^{\nabla}$ of a Hermitian connection $\nabla$ on $E$ are closely related: $\left[\frac{1}{2 \pi \sqrt{-1}} R \nabla\right]$ is the image of $c_{1}(E)$ under the natural map

$$
\iota: H^{2}(M, \mathbb{Z}) \rightarrow H^{2}(M, \mathbb{Z}) \otimes \mathbb{R} \simeq H_{d R}^{2}(M)
$$

Consequently the integral of the 2-form $\frac{1}{2 \pi \sqrt{-1}} R^{\nabla}$ over any smooth integral 2-cycle in $M$ has to be an integer. Hence the only symplectic forms that can be prequantized (that is, can occur as curvatures) are the forms whose integration over integral 2-cycles give integers. And if a symplectic form $\omega$ is integral (that is, the de Rham class [ $\omega$ ] lies in the image of the map $\iota$ above), the lift of $[\omega]$ to $H^{2}(M, \mathbb{Z})$ need not be unique. A solution to these problems (independently due to Kostant and to Souriau) can be stated as follows:

Theorem 3.6. Suppose the de Rham cohomology class of a closed 2-form $\sigma$ on a manifold $M$ lies in the image of $\iota: H^{2}(M, \mathbb{Z}) \rightarrow H_{d R}^{2}(M)$. Then there exists a Hermitian line bundle $E \rightarrow M$ with a Hermitian connection $\nabla$ such that $\frac{1}{2 \pi \sqrt{-1}} R^{\nabla}=\sigma$.

There is another solution to this problem that I find more satisfactory and to which Theorem 3.6 is a corollary. It has the additional merit of allowing one to prequantize orbifolds as well. It involves thinking of $\mathcal{D}(M)$ not just as a set but as a collection of objects in a category and upgrading the map (3.1) to a functor. The target of this functor is a category of differential cocycles: the objects of this category involve integral cocycles and differential forms. The functor will turn out to be an equivalence of categories. So it can be easily inverted (up to homotopy). We will take this up in the last section of the notes. In the mean time we proceed with prequantization.

Definition 3.7. Suppose $(E \rightarrow M,\langle\cdot, \cdot\rangle, \nabla)$ is a Hermitian line bundle with connection such that $\omega:=\frac{1}{2 \pi \sqrt{-1}} R^{\nabla}$ is symplectic. The prequantization is a linear map

$$
Q: C^{\infty}(M) \rightarrow \operatorname{Hom}(\Gamma(E), \Gamma(E)), \quad f \mapsto Q_{f}
$$

where the operator $Q_{f}$ is defined by

$$
Q_{f}(s)=\nabla_{\Xi_{f}} s-2 \pi \sqrt{-1} f \cdot s
$$

for all functions $f \in C^{\infty}(M)$ and all section $s \in \Gamma(E)$. Here, as before, $\Xi_{f}$ denotes the Hamiltonian vector field of $f$ with respect to the symplectic form $\omega$.

Remark 3.8. Our definition of $Q$ differs from a more traditional one by $\sqrt{-1}$. The physicists like to identify the Lie algebra of the unitary group with Hermitian matrices (and operators).

We next prove:

Lemma 3.9. The prequantization map $Q: C^{\infty}(M) \rightarrow \operatorname{Hom}(\Gamma(E), \Gamma(E))$ is a map of Lie algebras:

$$
\left[Q_{f}, Q_{g}\right] s=Q_{\{f, g\}} s
$$

for all sections $s \in \Gamma(E)$ and all functions $f, g \in C^{\infty}(M)$.

Proof. Since $\omega:=\frac{1}{2 \pi \sqrt{-1}} R^{\nabla}$, we have, by definition of curvature that

$$
\left(\left[\nabla_{X}, \nabla_{Y}\right]-\nabla_{[X, Y]}\right) s=2 \pi \sqrt{-1} \omega(X, Y) \cdot s
$$

for all vector fields $X, Y$ on $M$ and all sections $s \in \Gamma(E)$. Hence

$$
\left[\nabla_{\Xi_{f}}, \nabla_{\Xi_{g}}\right] s=\nabla_{\left[\Xi_{f}, \Xi_{g}\right]} s+2 \pi \sqrt{-1} \omega\left(\Xi_{f}, \Xi_{g}\right) \cdot s
$$

for all $f, g \in C^{\infty}(M)$. Since $\omega\left(\Xi_{f}, \Xi_{g}\right)=\{f, g\}$ and since $\left[\Xi_{f}, \Xi_{g}\right]=\Xi_{\{f, g\}}$ we get

$$
\left[\nabla_{\Xi_{f}}, \nabla_{\Xi_{g}}\right] s=\nabla_{\Xi_{\{f, g\}}} s+2 \pi \sqrt{-1}\{f, g\} s .
$$

Next observe that

$$
\begin{aligned}
Q_{f}\left(Q_{g} s\right) & =Q_{f}\left(\nabla_{\Xi_{g}} s-2 \pi \sqrt{-1} g \cdot s\right) \\
& =\nabla_{\Xi_{f}}\left(\nabla_{\Xi_{g}} s-2 \pi \sqrt{-1} g \cdot s\right)-2 \pi \sqrt{-1} f \cdot\left(\nabla_{\Xi_{g}} s-2 \pi \sqrt{-1} g \cdot s\right) \\
& =\nabla_{\Xi_{f}}\left(\nabla_{\Xi_{g}} s\right)-2 \pi \sqrt{-1} \Xi_{f}(g) \cdot s-2 \pi \sqrt{-1} g \nabla_{\Xi_{f}} s-2 \pi \sqrt{-1} f \nabla_{\Xi_{g}} s-4 \pi^{2} f g \cdot s
\end{aligned}
$$

Similarly,

$$
Q_{g}\left(Q_{f} s\right)=\nabla_{\Xi_{g}}\left(\nabla_{\Xi_{f}} s\right)-2 \pi \sqrt{-1} \Xi_{g}(f) \cdot s-2 \pi \sqrt{-1} f \nabla_{\Xi_{g}} s-2 \pi \sqrt{-1} g \nabla_{\Xi_{f}} s-4 \pi^{2} g \cdot f \cdot s .
$$


Hence

$$
\begin{aligned}
{\left[Q_{f}, Q_{g}\right] s } & =Q_{f}\left(Q_{g} s\right)-Q_{g}\left(Q_{f} s\right) \\
& =\left[\nabla_{\Xi_{f}}, \nabla_{\Xi_{g}}\right] s-2 \pi \sqrt{-1}(\{f, g\}-\{g, f\}) s \\
& =\left[\nabla_{\Xi_{f}}, \nabla_{\Xi_{g}}\right] s-4 \pi \sqrt{-1}\{f, g\} s \\
& =\nabla_{\Xi_{\{f, g\}}} s+2 \pi \sqrt{-1}\{f, g\} s-4 \pi \sqrt{-1}\{f, g\} s \quad \text { by }(3.2) \\
& =\nabla_{\Xi_{\{f, g\}}} s-2 \pi \sqrt{-1}\{f, g\} s=Q_{\{f, g\}} s .
\end{aligned}
$$

Definition 3.10. Let $(M, \omega)$ be a symplectic manifold of dimension $2 m$ and $(E \rightarrow M,\langle\cdot, \cdot)$,$\rangle be a Hermitian line$ bundle as before. A section $s \in \Gamma(E)$ is square integrable if the integral $\int_{M}\langle s, s\rangle \omega^{m}$ converges.

Clearly any section $s$ with compact support is square integrable. Moreover, for any two compactly supported sections $s, s^{\prime}$ of $E \rightarrow M$ the function $\left\langle s, s^{\prime}\right\rangle$ is compactly supported, hence the integral $\int_{M}\left\langle s, s^{\prime}\right\rangle \omega^{m}$ converges.

Notation 3.11. We denote the space of compactly supported sections of the bundle $E \rightarrow M$ by $\Gamma_{c}(E)$ :

$$
\Gamma_{c}(E):=\{s \in \Gamma(E) \mid \operatorname{supp}(s) \text { is compact }\} .
$$

The space $\Gamma_{c}(E)$ of compactly supported sections carries a natural Hermitian inner product defined by

$$
\left\langle\left\langle s, s^{\prime}\right\rangle\right\rangle=\int_{M}\left\langle s, s^{\prime}\right\rangle \omega^{m}
$$

for all $s, s^{\prime} \in \Gamma_{c}(E)$.

Definition 3.12. The prequantum Hilbert space associated with a prequantum line bundle $(E \rightarrow M,\langle\cdot, \cdot)$,$\rangle is the$ completion of the inner product space $\left(\Gamma_{c}(E),\langle\langle\cdot, \cdot\rangle\rangle\right)$ with the respect to the corresponding $L^{2}$ norm:

$$
\mathcal{H}_{0}:=\text { the completion of } \Gamma_{c}(E) \text {. }
$$

Lemma 3.13. The prequantization map $Q: C^{\infty}(M) \rightarrow \operatorname{Hom}\left(\Gamma_{c}(E), \Gamma_{c}(E)\right)$ is skew-Hermitian:

$$
\left\langle\left\langle Q_{f} s, s^{\prime}\right\rangle\right\rangle+\left\langle\left\langle s, Q_{f} s^{\prime}\right\rangle\right\rangle=0
$$

for all compactly supported sections $s, s^{\prime}$ of $E \rightarrow M$.

Proof. Observe that

$$
\left\langle 2 \pi \sqrt{-1} f s, s^{\prime}\right\rangle+\left\langle s, 2 \pi \sqrt{-1} f s^{\prime}\right\rangle=0
$$

for all functions $f \in C^{\infty}(M, \mathbb{R})$ and all square-integrable sections $s, s^{\prime}$. Next note that since the connection $\nabla$ is Hermitian we have

$$
\int_{M}\left\langle\nabla_{\Xi_{f}} s, s^{\prime}\right\rangle \omega^{m}+\int_{M}\left\langle s, \nabla_{\Xi_{f}} s^{\prime}\right\rangle \omega^{m}=\int_{M} \Xi_{f}\left\langle s, s^{\prime}\right\rangle \omega^{m} .
$$

Since the Lie derivative $\mathcal{L}_{\Xi_{f}} \omega$ of the symplectic form with respect to any Hamiltonian vector field $\Xi_{f}$ zero (see Lemma 2.11), we have $\mathcal{L}_{\Xi_{f}} \omega^{m}=0$ as well. Hence

$$
\mathcal{L}_{\Xi_{f}}\left(\left\langle s, s^{\prime}\right\rangle \omega^{m}\right)=\mathcal{L}_{\Xi_{f}}\left(\left\langle s, s^{\prime}\right\rangle\right) \omega^{m}+\left\langle s, s^{\prime}\right\rangle \mathcal{L}_{\Xi_{f}}\left(\omega^{m}\right)=\Xi_{f}\left\langle s, s^{\prime}\right\rangle \omega^{m}+0 .
$$

On the other hand by Cartan's magic formula, for any top degree form $\mu$ we have

$$
\mathcal{L}_{\Xi_{f}} \mu=\iota\left(\Xi_{f}\right) d \mu+d\left(\iota\left(\Xi_{f}\right) \mu\right)=d\left(\iota\left(\Xi_{f}\right) \mu\right),
$$

since $d \mu=0$. We conclude that

$$
\begin{aligned}
\int_{M}\left\langle\nabla_{\Xi_{f}} s, s^{\prime}\right\rangle \omega^{m}+\int_{M}\left\langle s, \nabla_{\Xi_{f}} s^{\prime}\right\rangle \omega^{m} & =\int_{M} \Xi_{f}\left\langle s, s^{\prime}\right\rangle \omega^{m} \\
& =\int_{M} \mathcal{L}_{\Xi_{f}}\left(\left\langle s, s^{\prime}\right\rangle \omega^{m}\right) \\
& =\int_{M} d\left(\imath\left(\Xi_{f}\right)\left\langle s, s^{\prime}\right\rangle \omega^{m}\right)=0
\end{aligned}
$$

where the last equality holds by Stokes' theorem. The result follows.

Remark 3.14. Note that the operators $Q_{f}$ are not bounded in the $L^{2}$ norm since they involve differentiation. So they do not extend to bounded operators on the completion $\mathcal{H}_{0}$. However, they are elliptic operators, and consequently extend to closed densely defined operators on $\mathcal{H}_{0}$. Not surprisingly their domain of definition consists of square integrable sections with square integrable (distributional) first derivatives. 


\section{Polarizations}

Recall that geometric prequantization associates to an integral symplectic manifold $(M, \omega)$ a Hilbert space $\mathcal{H}_{0}$ and to each real-valued function $f$ on $M$ a skew-Hermitian operator $Q_{f}: \mathcal{H}_{0} \rightarrow \mathcal{H}_{0}$. Unfortunately this is not correct physics.

Example 4.1. Suppose our classical configuration space is $\mathbb{R}$, the real line. This is the example with which we started these lectures. The corresponding classical phase space is the cotangent bundle $M=T^{*} \mathbb{R}$ with the canonical symplectic form $\omega=d p \wedge d q$. The corresponding prequantum line bundle $E$ is trivial: $E=T^{*} \mathbb{R} \times \mathbb{C} \rightarrow T^{*} \mathbb{R}$. Hence the prequantum Hilbert space is $\mathcal{H}_{0}$ is the space $L^{2}\left(T^{*} \mathbb{R}, \mathbb{C}\right)$ of complex valued square integrable functions. Quantum mechanics tells us that the correct Hilbert space consists of square-integrable functions of one variable $L^{2}(\mathbb{R}, \mathbb{C})$, not of functions of two variables.

A standard solution to this problem is to introduce a polarization. To define polarizations we start with linear algebra.

Definition 4.2. A Lagrangian subspace $L$ of a symplectic vector space $(V, \omega)$ is a subspace satisfying two conditions:

(1) $L$ is isotropic: $\omega\left(v, v^{\prime}\right)=0$ for all vectors $v, v^{\prime} \in L$;

(2) $L$ is maximally isotropic: for any isotropic subspace $L^{\prime}$ of $V$ containing $L$ we must have $L=L^{\prime}$.

Remark 4.3. A standard argument shows that if $L \subset(V, \omega)$ is Lagrangian then

$$
\operatorname{dim} L=\frac{1}{2} \operatorname{dim} V \text {. }
$$

Example 4.4. If $(V, \omega)=\left(\mathbb{R}^{2}, \omega=d p \wedge d q\right)$ then any line $L$ in $V$ is Lagrangian.

The analogous definition for submanifolds is as follows:

Definition 4.5. An immersed submanifold $L$ of a symplectic manifold $(M, \omega)$ is Lagrangian if $T_{x} L \subset\left(T_{x} M, \omega_{x}\right)$ is a Lagrangian subspace for each point $x \in L$.

Definition 4.6. A (real) polarization on a symplectic manifold $(M, \omega)$ is a subbundle $\mathcal{F} \subset T M$ of its tangent bundle such that

(1) $\mathcal{F}$ is Lagrangian: $\mathcal{F}_{x} \subset\left(T_{x} M, \omega_{x}\right)$ is a Lagrangian subspace for all points $x \in M$.

(2) $\mathcal{F}$ is integrable (or involutive): for all local sections $X, Y$ of $\mathcal{F} \rightarrow M$, the Lie bracket $[X, Y]$ is again a local section of $\mathcal{F}$. This conditions is often abbreviated as $[\mathcal{F}, \mathcal{F}] \subset \mathcal{F}$.

Remark 4.7. If $\mathcal{F} \subset T M$ is an integrable distribution, then by the Frobenius theorem there exists a foliation $\mathcal{L}_{\mathcal{F}}$ of $M$ tangent to the distribution $\mathcal{F}$. If in addition $\mathcal{F}$ is Lagrangian then the leaves of $\mathcal{L}_{\mathcal{F}}$ are immersed Lagrangian submanifolds of $M$.

Example 4.8. Suppose the symplectic manifold $M$ is a cotangent bundle of some manifold $N: M=T^{*} N$ with its standard symplectic form. Then $M$ has a polarization $\mathcal{F}$ given by the kernel of the differential of $\pi: T^{*} N \rightarrow N$ :

$$
\mathcal{F}=\operatorname{ker}(d \pi): T\left(T^{*} N\right) \rightarrow T N \text {. }
$$

In local coordinates $\left(q_{1}, \ldots, q_{n}, p_{1}, \ldots, p_{n}\right): T^{*} U \rightarrow \mathbb{R}^{n} \times \mathbb{R}^{n}(U \subset N$ open $)$ on $T^{*} N$ this polarization is given by

$$
\mathcal{F}=\left\{\sum_{i=1}^{n} a_{i} \frac{\partial}{\partial p_{i}} \mid a_{i} \in C^{\infty}(U)\right\} .
$$

The polarization $\mathcal{F}$ is called the vertical polarization of $T^{*} N$.

Example 4.9. Consider the punctured plane $M:=\mathbb{R}^{2} \backslash\{0\}$ with the symplectic form $d p \wedge d q$. The collection of circles $\left\{C_{r}:=\left\{(p, q) \in M \mid p^{2}+q^{2}=r^{2}\right\}\right\}_{r>0}$ forms a Lagrangian foliation of $M$. The tangent lines to the circle define a polarization $\mathcal{F}$ of $M$.

Remark 4.10. Real polarizations need not exist. Here is an example. It is not hard to show that any real line bundle over the two-sphere $S^{2}$ has to be trivial, hence has to have a nowhere zero section. Thus if $S^{2}$ has a polarization then it has a nowhere zero vector field, which contradicts a well-known theorem.

Since real polarization need not exist for interesting classical systems, one generalizes the notion of a real polarization to that of a complex polarization. A complex polarization on a symplectic manifold $(M, \omega)$ is a complex Lagrangian involutive subbundle of the complexified tangent bundle $T M \otimes \mathbb{C}$. We will not discuss them further. There is a well-developed theory of complex polarizations that an interested reader may consult.

Finally there are examples due to Mark Gotay [6] of symplectic manifolds that admit no polarizations whatsoever, real or complex. One can reconcile oneself to this fact by thinking that not all classical mechanical systems have quantum counterparts. 
Definition 4.11. Let $\mathcal{F}$ be a polarization on $(M, \omega)$ and $(\mathbb{L} \stackrel{\pi}{\rightarrow} M,\langle\cdot, \cdot\rangle, \nabla)$ a prequantum line bundle. A section $s \in \Gamma(\mathbb{L})$ is covariantly constant along $\mathcal{F}$ if $\nabla_{X} s=0$ for all sections $X \in \Gamma(\mathcal{F})$.

Notation 4.12. We denote the space of sections of the prequantum line bundle $\mathbb{L} \rightarrow M$ covariantly constant along a polarization $\mathcal{F}$ by $\Gamma_{\mathcal{F}}(\mathbb{L})$. Thus

$$
\Gamma_{\mathcal{F}}(\mathbb{L}):=\left\{s \in \Gamma(\mathbb{L}) \mid \nabla_{X} s=0 \text { for all } X \in \Gamma(\mathcal{F})\right\} .
$$

Remark 4.13. It is common to refer to the space $\Gamma_{\mathcal{F}}(\mathbb{L})$ as the space of polarized sections.

Example 4.14. If $M=T^{*} N, \mathbb{L}=T^{*} N \times \mathbb{C}$ and $\mathcal{F} \subset T M$ is the vertical polarization, then the space $\Gamma_{\mathcal{F}}(\mathbb{L}) \subset$ $C^{\infty}\left(T^{*} N, \mathbb{C}\right)$ consists of functions constant along the fibers of $\pi: T^{*} N \rightarrow N$. Thus $\Gamma_{\mathcal{F}}(\mathbb{L})=\pi^{*} C^{\infty}(N, \mathbb{C})$.

Example 4.15. Consider again the punctured plane $M=\mathbb{R}^{2} \backslash\{0\}$ with the polarization $\mathcal{F}$ defined by circles. We may take the trivial bundle $\mathbb{L}=M \times \mathbb{C} \rightarrow M$ as the prequantum line bundle. Its space of sections is simply the space of complex valued functions on $M$. The Hermitian inner product on $\mathbb{L}$ is defined by the standard Hermitian inner product on $\mathbb{C}$.

For any real-valued 1-form $\alpha$ on $M$ the map $\nabla: \Gamma(T M) \times C^{\infty}(M, \mathbb{C}) \rightarrow C^{\infty}(M, \mathbb{C})$ defined by

$$
\nabla_{X} f=X f+\sqrt{-1} \alpha(X) f
$$

is a Hermitian connection. Now consider the real-valued 1-form $\alpha$ on on $M$ given in polar coordinates $(r, \theta)$ by the equation $\alpha=r^{2} d \theta$. A section $f \in C^{\infty}(M, \mathbb{C})$ of $\mathbb{L}$ is covariantly constant along the polarization defined by the circles (cf. Example 4.9) if and only if

$$
\frac{\partial f}{\partial \theta}=-\sqrt{-1} r^{2} f
$$

A function $f$ solves the above equation if and only if it is of the form

$$
f(r, \theta)=g(r) e^{-\sqrt{-1} r^{2} \theta}
$$

for some function $g(r)$. Such an $f$ is a well-defined function on the punctured plane only if $r^{2} \in \mathbb{Z}$. Thus (4.1) has no nonzero smooth solutions.

Let us go back to the general situation: a prequantum line bundle $(\mathbb{L},\langle\cdot, \cdot\rangle, \nabla)$ over a symplectic manifold $(M, \omega)$ and a real polarization $\mathcal{F} \subset T M$. We now make several assumptions:

(1) The space of leaves $N:=M / \mathcal{F}$ is a Hausdorff manifold and the quotient map $\pi: M \rightarrow M / \mathcal{F} \equiv N$ is a submersion.

(2) The space of polarized sections $\Gamma_{\mathcal{F}}(\mathbb{L})$ is nonzero.

Remark 4.16. If we assume that the quotient map $\pi: M \rightarrow M / \mathcal{F}$ is proper then it places very severe restrictions on what the connected components of the leaves of $\mathcal{F}$ can be: they have to be compact tori. See Duistermaat [4], for example. In particular the fact that compact leaves of the polarization of a punctured plane turned out to be circles (i.e., one dimensional tori) should come as no surprise (q.v. Example 4.15). More generally the leaves of a Lagrangian fibration are open subsets of quotients of the form $V / \Gamma$ where $V$ is a finite dimensional real vector spaces and $\Gamma \subset V$ a discrete subgroup. That is, $V / \Gamma \simeq\left(\mathbb{R}^{k} / \mathbb{Z}^{k}\right) \times \mathbb{R}^{l}$ for some $k, l$ with $k+l=\operatorname{dim} V$.

The issue with existence of nonzero parallel sections reduces to the holonomy of the connection being trivial along the leaves. Since the curvature of the connection vanishes identically on each leaf, the obstruction to the existence of nonzero parallel (polarized) sections lies in the representations of the fundamental groups of the leaves. This is why it is not uncommon for the fundamental groups of the leaves to be assumed away. Fortunately there are examples of fibrations with simply connected leaves that are slightly more general than the cotangent bundles. They are the so-called "twisted cotangent bundles" and amount to the following. Let $Q$ be a manifold with an integral closed two-form $\tau$ (which may be degenerate) and let $M=T^{*} Q$. It is not hard to check that the two-form $\omega=\pi^{*} \tau+\omega_{T^{*} Q}$ is symplectic. Here $\pi: T^{*} Q \rightarrow Q$ is the canonical projection and $\omega_{T^{*} Q}$ is the canonical symplectic form on $T^{*} Q$ (q.v. Example 2.5). The Lagrangian foliation of $\left(T^{*} Q, \omega\right)$ is provided by the fibers of $\pi$, which are contractible.

Definition 4.17. Given a polarization $\mathcal{F} \subset T M$, the space of polarization preserving functions is the space $C_{\mathcal{F}}^{\infty}(M)$ defined by

$$
C_{\mathcal{F}}^{\infty}(M):=\left\{f \in C^{\infty}(M) \mid\left[\Xi_{f}, X\right] \in \Gamma(\mathcal{F}) \text { for all } X \in \Gamma(\mathcal{F})\right\},
$$

where, as before, $\Xi_{f}$ denotes the Hamiltonian vector field of the function $f$.

Remark 4.18. It is not hard to show that if $f$ is a polarization preserving function then the flow of its Hamiltonian vector field $\Xi_{f}$ preserves the leaves of the foliation defined by the distribution $\mathcal{F}$. 
Remark 4.19. If $\Xi_{f} \in \Gamma(\mathcal{F})$ then, since $\mathcal{F}$ is involutive, $f \in C_{\mathcal{F}}^{\infty}(M)$. Using again the fact that $\mathcal{F}$ is Lagrangian, it is not hard to show that if $f=\pi^{*} h$ for some $h \in C^{\infty}(M / \mathcal{F})$ then $\Xi_{f} \in \Gamma(\mathcal{F})$. In particular the space $C_{\mathcal{F}}^{\infty}(M)$ is non-trivial.

Lemma 4.20. The subspace $C_{\mathcal{F}}^{\infty}(M)$ of $C^{\infty}(M)$ is closed under the Poisson bracket.

Proof. Suppose $f, g \in C_{\mathcal{F}}^{\infty}(M)$ and $X \in \Gamma(\mathcal{F})$. Then

$$
\Xi_{\{f, g\}}=\left[\Xi_{f}, \Xi_{g}\right]
$$

Hence

$$
\left[X, \Xi_{\{f, g\}}\right]=\left[X,\left[\Xi_{f}, \Xi_{g}\right]\right]=\left[\left[X, \Xi_{f}\right], \Xi_{g}\right]+\left[\Xi_{f},\left[X, \Xi_{g}\right]\right]
$$

where the second equality hold by the Jacobi identity. Since $\left[X, \Xi_{f}\right],\left[X, \Xi_{g}\right] \in \Gamma(\mathcal{F})$ by assumption, and since $\Gamma(\mathcal{F})$ is a subspace of $\Gamma(T M)$ that is closed under brackets, we have $\left[X, \Xi_{\{f, g\}}\right] \in \Gamma(\mathcal{F})$.

Lemma 4.21. If $f \in C_{\mathcal{F}}^{\infty}(M)$ is a polarization-preserving function then the operator $Q_{f}$ defined by the prequantization map $Q: C^{\infty}(M) \rightarrow \operatorname{End}(\Gamma(\mathbb{L}))$ preserves the space $\Gamma_{\mathcal{F}}(\mathbb{L})$ of $\mathcal{F}$ polarized sections, the sections covariantly constant along $\mathcal{F}$.

Hence we get a map of Lie algebras

$$
Q: C_{\mathcal{F}}^{\infty}(M) \rightarrow \operatorname{End}\left(\Gamma_{\mathcal{F}}(\mathbb{L})\right)
$$

Proof. Note first that for any vector field $X \in \Gamma(T M)$ and any function $f \in C^{\infty}(M)$

$$
X(f)=d f(X)=\left(\iota\left(\Xi_{f}\right) \omega\right)(X)=\omega\left(X, \Xi_{f}\right) .
$$

To prove the lemma we need to show that

$$
\nabla_{X}\left(Q_{f} s\right)=0
$$

for all vector fields $X \in \Gamma(\mathcal{F})$, all functions $f \in C_{\mathcal{F}}^{\infty}(M)$ and all polarized sections $s \in \Gamma_{\mathcal{F}}(\mathbb{L})$. Now

$$
\nabla_{X}\left(\nabla_{\Xi_{f}} s-2 \pi \sqrt{-1} f s\right)=\nabla_{X}\left(\nabla_{\Xi_{f}} s\right)-2 \pi \sqrt{-1} X(f) s-2 \pi \sqrt{-1} f \nabla_{X} s .
$$

Note that by assumption $2 \pi \sqrt{-1} f \nabla_{X} s=0$. By definition of curvature,

$$
\nabla_{X}\left(\nabla_{\Xi_{f}} s\right)=\nabla_{\Xi_{f}}\left(\nabla_{X} s\right)+\nabla_{\left[X, \Xi_{f}\right]} s+R^{\nabla}\left(X, \Xi_{f}\right) s .
$$

By assumption on $X, f$ and $s$, the first two terms are 0 . Also, by definition of the connection

$$
R^{\nabla}=2 \pi \sqrt{-1} \omega \text {. }
$$

We conclude that

Putting it all together we see that

$$
\nabla_{X}\left(\nabla_{\Xi_{f}} s\right)=0+0+2 \pi \sqrt{-1} \omega\left(X, \Xi_{f}\right) s
$$

$$
\nabla_{X}\left(Q_{f} s\right)=2 \pi \sqrt{-1} \omega\left(X, \Xi_{f}\right) s-2 \pi \sqrt{-1} X(f) s=2 \pi \sqrt{-1}\left(\omega\left(X, \Xi_{f}\right)-\omega\left(X, \Xi_{f}\right)\right) s=0 .
$$

We would now like to define an inner product on the space $\Gamma_{\mathcal{F}}(\mathbb{L})$ of polarized sections. If the fibers of $\pi: M \rightarrow$ $M / \mathcal{F}$ are compact, then as before we can define an inner product on a subspace of $\Gamma_{\mathcal{F}}(\mathbb{L})$ consisting of square integrable sections - c.f. Definition 3.10 and the subsequent discussion. The completion of this space would give us the desired Hilbert space. However, as we have seen in Example 4.15, the space of (smooth) polarized section can be 0 . This is not just an accident of the particular example, but is fairly typical, since fibers of proper Lagrangian fibrations are tori. The solution to this problem - the lack of nonzero smooth polarized sections - is to consider distributional polarized sections. We will not say anything further on this topic in these notes. A curious reader may consult the discussion of distributional sections in [12].

If the leaves of the polarization on a symplectic manifold $(M, \omega)$ are not compact (as is the case of the vertical polarization on a cotangent bundle $T^{*} N$ ) then none of the polarized sections are square integrable with respect to the symplectic volume form $\omega^{m}\left(m=\frac{1}{2} \operatorname{dim} M\right)$. On the other hand, the Hermitian inner product $\left\langle s, s^{\prime}\right\rangle$ of two polarized sections $s, s^{\prime} \in \Gamma_{\mathcal{F}}(\mathbb{L})$ is constant along the fibers of the submersion $\pi: M \rightarrow M / \mathcal{F}$, hence descends to a function on the leaf space $M / \mathcal{F}$. Thus it is tempting to push the function $\left\langle s, s^{\prime}\right\rangle$ down to $M / \mathcal{F}$ and integrate it over the leaf space. The problem is that the leaf space $M / \mathcal{F}$ has no preferred measure or volume. For instance suppose $(M, \omega)$ is a 2-dimensional symplectic vector space $\left(V, \omega_{V}\right)$. Here we think of $\omega_{V}$ as a constant coefficient differential form. Then any line $\ell \subset V$ defines a polarization whose space of leaves is the quotient vector space $V / \ell$. While the vector space $V / \ell$ is one dimensional in this example, and thus isomorphic to the real line $\mathbb{R}$, there is no preferred identification of $V / \ell$ with $\mathbb{R}$ and no preferred measure on $V / \ell$. 
Let us recap where we are. We have an integral symplectic manifold $(M, \omega)$, a prequantum line bundle $\mathbb{L} \rightarrow M$ with connection $\nabla$ whose curvature is $2 \pi \sqrt{-1} \omega$, a Lagrangian foliation $\mathcal{F}$ of $M$ so that the space of leaves $M / \mathcal{F}$ is a Hausdorff manifold, the quotient map $\pi: M \rightarrow M / \mathcal{F}$ is a fibration and the holonomy representation of the fundamental groups of the leaves with respect to the connection $\nabla$ are trivial. In this case the prequantum line bundle $\mathbb{L} \rightarrow M$ descends to a Hermitian line bundle $\mathbb{L} / \mathcal{F} \rightarrow M / \mathcal{F}$. We now consider the new complex line bundle $\mathbb{L} / \mathcal{F} \otimes|T(M / \mathcal{F})|^{1 / 2}$, the bundle $\mathbb{L} / \mathcal{F}$ twisted by the bundle of half densities on $M / \mathcal{F}$ (q.v. Definition B.11). We have an isomorphism

$$
\Gamma\left(\mathbb{L} / \mathcal{F} \otimes|T(M / \mathcal{F})|^{1 / 2}\right) \simeq \Gamma(\mathbb{L} / \mathcal{F}) \otimes_{C^{\infty}(M / \mathcal{F})} \Gamma\left(|T(M / \mathcal{F})|^{1 / 2}\right)
$$

of $C^{\infty}(M / \mathcal{F})$ modules.

It makes sense to talk about the sections of $\mathbb{L} / \mathcal{F} \otimes|T(M / \mathcal{F})|^{1 / 2}$ being square integrable, and it makes sense to define a sesquilinear pairing of two square integrable sections. This is done as follows. As we observed in Remark B.14, the bundle $|T(M / \mathcal{F})|^{1 / 2}$ of half densities is trivial, that is, it has a nowhere zero global section. Hence any section of $\mathbb{L} / \mathcal{F} \otimes|T(M / \mathcal{F})|^{1 / 2}$ is of the form $s \otimes \mu$ where $s \in \Gamma(\mathbb{L} / \mathcal{F}) \simeq \Gamma_{\mathcal{F}}(\mathbb{L})$ and $\mu$ is a $1 / 2$ density on $M / \mathcal{F}$. Now given a polarized section $s \in \Gamma_{\mathcal{F}}(\mathbb{L})$ and a $1 / 2$ density $\mu$ on $M / \mathcal{F}$ we can form a 1 density

$$
\langle s, s\rangle \bar{\mu} \mu=\|s\|^{2}|\mu|^{2}
$$

on $M / \mathcal{F}$. Moreover the map

$$
(\mathbb{L} / \mathcal{F}) \otimes_{C^{\infty}(M / \mathcal{F})} \Gamma\left(|T(M / \mathcal{F})|^{1 / 2}\right) \rightarrow|M / \mathcal{F}|^{1}, \quad s \otimes \mu \mapsto\|s\|^{2}|\mu|^{2}
$$

is well-defined. Here, as in Appendix B, $|M / \mathcal{F}|^{1}$ denotes the space of 1-densities on the manifold $M / \mathcal{F}$, which is the space of sections of the bundle $|T(M / \mathcal{F})|^{1}$ of 1-densities. It is not hard to show that the space of compactly supported polarized sections of the twisted prequantum line bundle forms a vector space with a Hermitian inner product given by

$$
\left\langle\left\langle s_{1} \otimes \mu_{1}, s_{2} \otimes \mu_{2}\right\rangle\right\rangle:=\int_{M / \mathcal{F}}\left\langle s_{1}, s_{2}\right\rangle \bar{\mu}_{1} \mu_{2} .
$$

The completion of this complex vector space with respect to $\langle\langle\cdot, \cdot\rangle\rangle$ is the intrinsic quantum space associate with the data $(\mathbb{L} \rightarrow M, \nabla, \mathcal{F},\langle\cdot, \cdot\rangle)$. A bit more effort gives a representation of the Lie algebra $C_{\mathcal{F}}^{\infty}(M)$ on this quantum space. See [13],[12] or [16].

\section{Prequantization of Differential cocycles}

Let $L \stackrel{\pi}{\rightarrow} M$ be a complex line bundle, $\langle\cdot, \cdot\rangle$ a Hermitian inner product and $\nabla$ a Hermitian connection. In Lecture 1 we were trying to find a section of the curvature map

$$
\text { curv }:(L \stackrel{\pi}{\rightarrow} M,\langle\cdot, \cdot\rangle, \nabla) \longmapsto \frac{1}{\sqrt{-1}} R^{\nabla} \in \Omega^{2}(M),
$$

which is neither 1-1 nor onto. Recall that a Hermitian line bundle with connection over a manifold $M$ defines a principal $S^{1}$ bundle with a connection 1-form and conversely. Since 1-forms obviously pull back, it will be convenient for us to replace the problem of finding the section of (5.1) by the problem of finding a section of

$$
\text { curv }:\left(S^{1} \rightarrow P \rightarrow M, A \in \Omega^{1}(P, \mathbb{R})^{S^{1}}\right) \rightarrow F_{A} \in \Omega^{2}(M, \mathbb{R}),
$$

where $F_{A}$ denotes the curvature of the connection 1-form $A$. Note that here we think of the circle $S^{1}$ as $\mathbb{R} / \mathbb{Z}$ and not as the group $U(1)$ of unit complex numbers. Hence now our connection 1-forms are $\mathbb{R}$-valued.

For a given manifold $M$, the collection of all principal $S^{1}$-bundles with connection 1 -forms over $M$ forms a category, which we will denote by $D B S^{1}(M) .{ }^{1}$ More precisely the objects of the category $D B S^{1}(M)$ are pairs $(P, A)$, where $P$ is a principal $S^{1}$ bundle over $M$ and $A \in \Omega^{1}(P)$ is a connection 1-form on $P$. Given two objects $(P, A),\left(P^{\prime}, A^{\prime}\right)$ of $D B S^{1}(M)$ the set $\operatorname{Hom}\left((P, A),\left(P^{\prime}, A^{\prime}\right)\right)$ of morphisms between them is defined by

$$
\operatorname{Hom}\left((P, A),\left(P^{\prime}, A^{\prime}\right)\right)=\left\{\phi: P \rightarrow P^{\prime} \mid \phi \text { is } S^{1} \text { equivariant, } \phi \text { induces identity on } M, \phi^{*} A^{\prime}=A\right\} \text {. }
$$

Notice that all morphisms are invertible, so the category $D B S^{1}(M)$ is a groupoid by definition (see Appendix A).

Our solution of non-invertability of the curvature map proceeds along the following lines. We will construct a category $\mathcal{D C}(M)$ of differential cocycles, so that:

(1) The objects of $\mathcal{D C}(M)$ involve differential forms.

(2) There is an equivalence of categories $D B S^{1}(M) \stackrel{D C h}{\longrightarrow} \mathcal{D C}(M)$.

\footnotetext{
${ }^{1}$ This is not a standard notation. The $B S^{1}(M)$ is supposed to remind the reader of the classifying space $B S^{1}$, maps into which classify principal $S^{1}$ bundles. The $D$ stands for "differential," i.e., the connection.
} 
Since equivalences of categories are invertible (up to natural isomorphisms) this will achieve our objective. ${ }^{2}$ The construction of the category $\mathcal{D C}(M)$ was carried out as a toy example in a paper by Hopkins and Singer [9], which is where we copy the definition from. In constructing the functor $D C h$ we will follow [11]. The construction of $\mathcal{D C}$ requires several steps.

Step 1: Categories from cochain complexes. Let $A^{\bullet}=\left\{A^{\bullet} \stackrel{d}{\rightarrow} A^{\bullet+1}\right\}$ be a cochain complex of abelian groups. For example, $A^{\bullet}=\Omega^{\bullet}(M)$, the complex of differential forms on a manifold $M$. For each index $n \geq 0$ there is a category $\mathcal{H}^{n}\left(A^{\bullet}\right)$ with the set $\left\{z \in A^{n} \mid d z=0\right\}$ of cocycles of degree $n$ being its set of objects. The set of morphisms $\operatorname{Hom}\left(z, z^{\prime}\right)$ for two cocycles $z, z^{\prime}$ is defined by

$$
\operatorname{Hom}\left(z, z^{\prime}\right)=\left\{(z,[b]) \in \operatorname{ker}\left(d: A^{n} \rightarrow A^{n+1}\right) \times A^{n-1} / d A^{n-2} \mid z^{\prime}=z+d b\right\} \simeq\left\{[b] \in A^{n-1} / d A^{n-2} \mid z^{\prime}=z+d b\right\}
$$

The composition of morphisms is addition + :

$$
\left(z^{\prime},\left[b^{\prime}\right]\right) \circ(z,[b])=\left(z,\left[b+b^{\prime \prime}\right]\right) .
$$

The category $\mathcal{H}^{n}\left(A^{\bullet}\right)$ is a groupoid with the set $\pi_{0}\left(\mathcal{H}^{n}\left(A^{\bullet}\right)\right.$ of equivalence classes of objects being the cohomology group $H^{n}\left(A^{\bullet}\right)$. The category $\mathcal{H}^{n}\left(A^{\bullet}\right)$ may also be viewed as an action groupoid for the action of $A^{n-1} / d A^{n-2}$ on $\operatorname{ker}\left(d: A^{n} \rightarrow A^{n+1}\right)$ by way of $d: A^{n-1} \rightarrow A^{n}$.

Next suppose we have a contravariant functor from the category Man of manifolds and smooth maps to the category CoChain of cochain complexes (such a functor is often called a presheaf of cochain complexes):

$$
A^{\bullet}: \operatorname{Man}^{o p} \rightarrow \text { CoChain, } \quad M \mapsto A^{\bullet}(M) .
$$

An example to keep in mind is the functor that assigns to each manifold the complex of differential forms and to a map of manifolds the pullback of differential forms. Then each smooth map $f: M \rightarrow N$ between manifolds induces a functor

$$
\mathcal{H}^{n}(f): \mathcal{H}^{n}\left(A^{\bullet}(N)\right) \rightarrow \mathcal{H}^{n}\left(A^{\bullet}(M)\right)
$$

with

$$
z^{\prime} \underset{(z,[b])}{\longleftarrow} \longmapsto \quad\left(f^{*} z^{\prime} \underset{\left(f^{*} z,\left[f^{*} b\right]\right)}{\longleftarrow} f^{*} z\right)
$$

Step 2: The presheaf of differential cocycles. We need to introduce more notation. Denote by $C^{\bullet}(M, \mathbb{Z})$ the complex of $\left(C^{\infty}\right)$ singular integral cochains on a manifold $M$ and by $C^{\bullet}(M, \mathbb{R})$ the complex of $\left(C^{\infty}\right)$ singular real-valued cochains. We have maps of complexes

$$
C^{\bullet}(M, \mathbb{Z}) \rightarrow C^{\bullet}(M, \mathbb{R}) \quad \text { and } \quad \Omega^{\bullet}(M) \rightarrow C^{\bullet}(M, \mathbb{R})
$$

The second map sends a differential $k$-form $\sigma$ to a functional on the space of real $k$-chains: the value of this functional on a chain $s$ is the integral $\int_{s} \sigma$. We would like to find a complex $D C^{\bullet}(M)$ that fits into the diagram

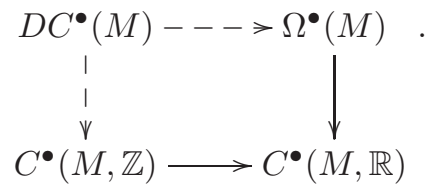

We define this complex as follows:

$$
D C^{k}(M)=\left\{(c, h, \omega) \in C^{k}(M, \mathbb{Z}) \times C^{k-1}(M, \mathbb{R}) \times \Omega^{k}(M) \mid \omega=0 \text { for } k<2\right\}
$$

with the differential $\tilde{d}$ defined by

$$
\tilde{d}(c, h, \omega)=(\delta c, \omega-c-\delta h, d \omega) .
$$

Note that in defining $\tilde{d}$ we suppressed the maps $C^{\bullet}(M, \mathbb{Z}) \rightarrow C^{\bullet}(M, \mathbb{R})$ and $\Omega^{\bullet}(M) \rightarrow C^{\bullet}(M, \mathbb{R})$. In particular, when we think of a differential $k$-form $\tau$ as a real cochain, we write its differential as $\delta \tau$. That is, for any $k+1$ chain $\gamma$ we have

$$
\delta \tau(\gamma)=\int_{\gamma} d \tau
$$

It is not hard to show that

$$
\tilde{d} \circ \tilde{d}=0
$$

Indeed,

$$
\tilde{d}(\tilde{d}(c, h, \omega))=\tilde{d}(\delta c, \omega-c-\delta h, d \omega)=\left(\delta^{2} c, d \omega-\delta c-\delta(\omega-c-\delta h), d^{2} \omega\right)=(0,0,0) .
$$

\footnotetext{
${ }^{2} \mathrm{~A}$ reader who is not fluent in category theory may wish at this point to contemplate Example A.23 of two rather different looking but equivalent categories.
} 
We are now ready to define the category $\mathcal{D C}(M)$ of differential cocycles by setting

$$
\mathcal{D C}(M):=\mathcal{H}^{2}\left(D C^{\bullet}(M)\right) .
$$

By construction the set of objects $\mathcal{D C}(M)_{0}$ of this category is

$$
\mathcal{D C}(M)_{0}=\left\{(c, h, \omega) \in C^{2}(M, \mathbb{Z}) \times C^{1}(M, \mathbb{R}) \times \Omega^{2}(M) \mid \delta c=0, d \omega=0, \omega=c-\delta h\right\}
$$

and the morphisms are defined by

$$
\operatorname{Hom}\left((c, h, \omega),\left(c^{\prime}, h^{\prime}, \omega^{\prime}\right)\right)=\left\{[e, k, 0] \mid e \in C^{1}(M, \mathbb{Z}), k \in C^{0}(M, \mathbb{R}) \text { and } \begin{array}{c}
c^{\prime}-c=\delta e \\
h^{\prime}-h=-\delta k-e \\
\omega-\omega^{\prime}=0
\end{array}\right\} .
$$

The following theorem then holds (it is presented as a warm-up example in [9]):

Theorem 5.1. For each manifold $M$ there exists an equivalence of categories

$$
D C h_{M}: D B S^{1}(M) \rightarrow \mathcal{D C}(M)
$$

with $D C h_{M}(P, A)=\left(c(P, A), h(P, A), F_{A}\right)$ for each principal circle bundle with connection $(P, A)$. Here as before $F_{A}$ denotes the curvature of a connection $A$.

Remark 5.2. The functor that assigns a prequantum line bundle to a differential cocycle is the "homotopy inverse" of the functor $D C h_{M}$.

Finally here is an outline of an argument as to why this theorem is true and how you would go about writing down the functor $D C h$. I will be following the presentation in [11]. Here are the main ideas:

(1) Do it for all manifolds at once.

(2) Restate the theorem for presheaves of categories:

There exists a morphism $D C h_{(\cdot)}: D B S^{1}(\cdot) \rightarrow \mathcal{D C}(\cdot)$ of presheaves of categories with the desired properties.

Here the $\cdot$ is a place holder for a manifold.

(3) Write down the functor $D C h$ explicitly for the sub-presheaf of trivial bundles $D B S_{\text {triv }}^{1}(\cdot) \subset D B S^{1}(\cdot)$. This is not hard. The set of objects of the presheaf $D B S_{\text {triv }}^{1}(\cdot)$ on a manifold $M$ is the set

$$
D B S_{\text {triv }}^{1}(M)_{0}=\left\{\left(M \times S^{1}, a+d \theta\right) \mid a \in \Omega^{1}(M)\right\},
$$

The set of morphisms is

$$
\operatorname{Hom}\left(\left(M \times S^{1}, a+d \theta\right),\left(M \times S^{1}, a^{\prime}+d \theta\right)\right)=\left\{f: M \rightarrow S^{1} \mid a=a^{\prime}+f^{*} d \theta\right\} .
$$

For each manifold $M$ we therefore define the functor $D C h_{M}: D B S_{\text {triv }}^{1}(M) \rightarrow \mathcal{D C}(M)$ as follows: on objects

$$
D C h_{M}\left(M \times S^{1}, a+d \theta\right)=(0, a, d a),
$$

on morphisms

$$
D C h_{M}\left(f: M \rightarrow S^{1}\right)=\left[\delta(\tilde{f})-f^{*} d \theta, \tilde{f}, 0\right],
$$

where $\tilde{f}: M \rightarrow \mathbb{R}$ is any lift of $f$ (not necessarily continuous). We think of $\tilde{f}$ as a real 0 -cochain:

$$
\tilde{f}\left(\sum_{p \in M} n_{p}\right)=\sum n_{p} \tilde{f}(p)
$$

for any zero chain $\sum_{p \in M} n_{p}$.

The rest of the argument uses the following facts (see [11] for details):

(1) The functor $D C h_{M}: D B S_{\text {triv }}^{1}(M) \rightarrow \mathcal{D C}(M)$ is bijective on Hom's (that is, it is a fully faithful functor).

(2) If $M$ is contractible (e.g. an open ball) then $D C h_{M}$ is essentially surjective. Of course, for a general manifold $M$ not all bundles over $M$ are trivial, but they are all locally trivial.

(3) Any bundle with a connection can be glued together out of the trivial ones.

(4) Differential cochains glue like bundles. Showing this requires work.

These last two facts amount to saying that the two presheaves $D B S^{1}(\cdot)$ and $\mathcal{D C}(\cdot)$ are stacks over $\underline{\text { Man }}$, the category of Manifolds.

The presheaf $D B S_{\text {triv }}^{1}$ is not a stack since gluing a bunch of trivial bundles together need not result in a trivial bundle. There is an operation on presheaves of categories called stackification. This is a version of sheafification 
for sheaves of categories. The stackification of $D B S_{\text {triv }}^{1}$, not surprisingly, is $D B S^{1}$. Therefore, by the universal property of stackifications there is a unique functor $D C h: D B S^{1}(\cdot) \rightarrow \mathcal{D C}(\cdot)$ making the following diagram

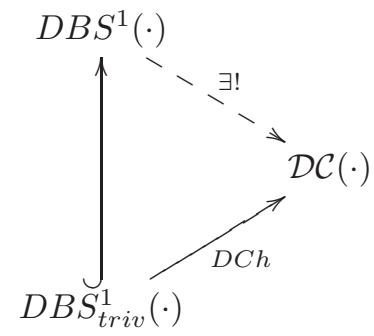

commute. The resulting functor

$$
D C h: D B S^{1}(\cdot) \rightarrow \mathcal{D C}(\cdot)
$$

is an equivalence of categories since it is an equivalence of categories locally.

\section{Appendix A. Elements of CAtegory theory}

\section{A.1. Basic notions.}

We start by recalling the basic definitions of category theory, mostly to fix our notation. This appendix may be useful to the reader with some background in category theory; the reader with little to no experience in category theory may wish to consult a textbook such as [2].

Definition A.1 (Category). A category A consists of

(1) A collection ${ }^{3} A_{0}$ of objects;

(2) For any two objects $a, b \in \mathrm{A}_{0}$, a set $\operatorname{Hom}_{\mathrm{A}}(a, b)$ of of morphisms (or arrows);

(3) For any three objects $a, b, c \in \mathrm{A}_{0}$, and any two arrows $f \in \operatorname{Hom}_{\mathrm{A}}(a, b)$ and $g \in \operatorname{Hom}_{\mathrm{A}}(b, c)$, a composite $g \circ f \in \operatorname{Hom}_{\mathrm{A}}(a, c)$, i.e., for all triples of objects $a, b, c \in \mathrm{A}_{0}$ there is a composition map

$$
\begin{gathered}
\circ: \operatorname{Hom}_{\mathrm{A}}(b, c) \times \operatorname{Hom}_{\mathrm{A}}(a, b) \rightarrow \operatorname{Hom}_{\mathrm{A}}(a, c), \\
\operatorname{Hom}_{\mathrm{A}}(b, c) \times \operatorname{Hom}_{\mathrm{A}}(a, b) \ni(g, f) \mapsto g \circ f \in \operatorname{Hom}_{\mathrm{A}}(a, c) .
\end{gathered}
$$

This composition operation is associative and has units, that is,

i. for any triple of morphisms $f \in \operatorname{Hom}_{\mathrm{A}}(a, b), g \in \operatorname{Hom}_{\mathrm{A}}(b, c)$ and $h \in \operatorname{Hom}_{\mathrm{A}}(c, d)$ we have

$$
h \circ(g \circ f)=(h \circ g) \circ f ;
$$

ii. for any object $a \in \mathrm{A}_{0}$, there exists a morphism $1_{a} \in \operatorname{Hom}_{\mathrm{A}}(a, a)$, called the identity, which is such that for any $f \in \operatorname{Hom}_{\mathrm{A}}(a, b)$ we have

$$
f=f \circ 1_{a}=1_{b} \circ f .
$$

We denote the collection of all morphisms of a category $A$ by $A_{1}$ :

$$
\mathrm{A}_{1}=\bigsqcup_{a, b \in \mathrm{A}_{0}} \operatorname{Hom}_{\mathrm{A}}(a, b) .
$$

Remark A.2. The symbol "o" is customarily suppressed in writing out compositions of two morphisms. Thus

$$
g f \equiv g \circ f .
$$

Example A.3 (Category Set of sets). The collection Set of all sets forms a category. The objects of Set are sets, the arrows of Set are ordinary maps and the composition of arrows is the composition of maps.

Example A.4 (Category Vect of vector spaces). The collection Vect of all real vector spaces (not necessarily finite dimensional) forms a category. Its objects are vector spaces and its morphisms are linear maps. The composition of morphisms is the ordinary composition of linear maps.

Example A.5 (The category Mat of coordinate vector spaces). The objects of this category are coordinate vector spaces $0=\mathbb{R}^{0}, \mathbb{R}^{1}, \ldots, \mathbb{R}^{n} \ldots$. The set of morphism from $\mathbb{R}^{m}$ to $\mathbb{R}^{n}$ is the set of all $n \times m$ matrices. The composition of morphisms is given by a matrix multiplication.

\footnotetext{
${ }^{3} \mathrm{~A}$ collection may be too big to be a set; we will ignore the set-theoretic issues this may lead to.
} 
Remark A.6. For a category $A$ there are two maps from the collection $A_{1}$ of its arrows to the collection $A_{0}$ of objects called source and target and denoted respectively by $s$ and $t$. They are defined by requiring that

$$
s(f)=a \text { and } t(f)=b \text { for any } f \in \operatorname{Hom}_{\mathrm{A}}(a, b) .
$$

Definition A.7. A subcategory A of a category B is a collection of some objects $A_{0}$ and some arrows $A_{1}$ of $B$ such that:

- For each object $a \in \mathrm{A}_{0}$, the identity $1_{a}$ is in $\mathrm{A}_{1}$;

- For each arrow $f \in \mathrm{A}_{1}$ its source and target $s(f), t(f)$ are in $\mathrm{A}_{0}$;

- for each pair $(f, g) \in \mathrm{A}_{0} \times \mathrm{A}_{0}$ of composable arrows $a \stackrel{f}{\rightarrow} a^{\prime} \stackrel{g}{\rightarrow} a^{\prime \prime}$ the composite $g \circ f$ is in $\mathrm{A}_{1}$ as well.

Remark A.8. Naturally a subcategory is a category in its own right.

Example A.9. The collection FinSet of all finite sets and all maps between them is a subcategory of Set hence a category. The collection FinVect of real finite dimensional vector spaces and linear maps is a subcategory of Vect.

Example A.10. A subcategory FinVect ${ }^{i s o}$ is defined to have the same objects as the category of FinVect. Its morphisms are isomorphisms of vector spaces. Since the composition of two linear isomorphisms is an isomorphism FinVect ${ }^{i s o}$ is a subcategory of FinVect.

Note that for any object $V$ in FinVect ${ }^{i s o}$, that is, for any finite dimensional vector space $V$, the set of morphisms $\operatorname{Hom}(V, V)$ in FinVect ${ }^{i s o}$ is $G L(V)$, the Lie group of invertible linear maps from $V$ to $V$.

Compare this to the fact that in the category FinVect the set of morphisms $\operatorname{Hom}(V, V)$ is $\operatorname{End}(V)$, the space of all linear maps from $V$ to itself.

Definition A.11 (isomorphism). An arrow $f \in \operatorname{Hom}_{\mathrm{A}}(a, b)$ in a category $\mathrm{A}$ is an isomorphism if there is an arrow $g \in \operatorname{Hom}_{A}(b, a)$ with $g \circ f=1_{a}$ and $f \circ g=1_{b}$. We think of $f$ and $g$ as inverses of each other and may write $g=f^{-1}$. Clearly $g=f^{-1}$ is also an isomorphism.

Two objects $a, b \in \mathrm{A}_{0}$ are isomorphic if there is an isomorphism $f \in \operatorname{Hom}_{\mathrm{A}}(a, b)$. We will also say that $a$ is isomorphic to $b$.

Definition A.12 (Groupoid). A groupoid is a category in which every arrow is an isomorphism.

Example A.13. The category FinVect ${ }^{i s o}$ is a groupoid.

Definition A.14 (Functor). A (covariant) functor F : A $\rightarrow$ B from a category A to a category B is a map on the objects and arrows of $\mathrm{A}$ such that every object $a \in \mathrm{A}_{0}$ is assigned an object $F a \in \mathrm{B}_{0}$, every arrow $f \in \operatorname{Hom}_{\mathrm{A}}(a, b)$ is assigned an arrow $F f \in \operatorname{Hom}_{\mathrm{B}}(F a, F b)$, and such that composition and identities are preserved, namely

$$
F(f \circ g)=F f \circ F g, \quad F 1_{a}=1_{F a} .
$$

A contravariant functor $G$ from A to $\mathrm{B}$ is a map on the objects and arrows of A such that every object $a \in \mathrm{A}_{0}$ is assigned an object $G a \in \mathrm{B}_{0}$, every arrow $f \in \operatorname{Hom}_{\mathrm{A}}(a, b)$ is assigned an arrow $G f \in \operatorname{Hom}_{\mathrm{B}}(G b, G a)$ (note the order reversal), such that identities are preserved, and the composition of arrows is reversed:

$$
G(f \circ g)=G(g) \circ G(f)
$$

for all composable pairs of arrows $f, g$ of A.

Example A.15. There is a natural functor $\iota:$ Mat $\rightarrow$ FinVect which is the identity on objects and maps an $n \times m$ matrix to the corresponding linear map.

Example A.16. The functor $(-)^{*}:$ FinVect $\rightarrow$ FinVect that takes the duals, that is, $(V \stackrel{A}{\longrightarrow} W) \mapsto\left(V^{*} \stackrel{A^{*}}{\longleftarrow} W^{*}\right)$ is a contravariant functor.

Remark A.17. Since functors are maps, functors can be composed.

Definition A.18. A functor $F: \mathrm{A} \rightarrow \mathrm{B}$ is

(1) full if $F: \operatorname{Hom}_{\mathrm{A}}\left(a, a^{\prime}\right) \rightarrow \operatorname{Hom}_{\mathrm{B}}\left(F a, F a^{\prime}\right)$ is surjective for all pairs of objects $a, a^{\prime} \in \mathrm{A}_{0}$;

(2) faithful if $F: \operatorname{Hom}_{\mathrm{A}}\left(a, a^{\prime}\right) \rightarrow \operatorname{Hom}_{\mathrm{B}}\left(F a, F a^{\prime}\right)$ is injective for all pairs of objects $a, a^{\prime} \in \mathrm{A}_{0}$

(3) fully faithful if $F: \operatorname{Hom}_{\mathrm{A}}\left(a, a^{\prime}\right) \rightarrow \operatorname{Hom}_{\mathrm{B}}\left(F a, F a^{\prime}\right)$ is a bijection for all pairs of objects $a, a^{\prime} \in \mathrm{A}_{0}$;

(4) essentially surjective if for any object $b \in \mathrm{B}_{0}$ there is an object $a \in \mathrm{A}_{0}$ and an isomorphism $f \in \operatorname{Hom}_{\mathrm{B}}(F(a), b)$. That is, for any object $b$ of $\mathrm{B}$ there is an object $a$ of $\mathrm{A}$ so that $b$ and $F(a)$ are isomorphic.

Example A.19. The functor $\iota:$ Mat $\rightarrow$ FinVect is fully faithful (since any linear map from $\mathbb{R}^{n}$ to $\mathbb{R}^{m}$ is uniquely determined by what it does on the standard basis) and essentially surjective (since any real vector space of dimension $n$ is isomorphic to $\mathbb{R}^{n}$ ). 
Definition A.20 (Natural Transformation). Let $F, G: \mathrm{A} \rightarrow$ B be a pair of functors. A natural transformation $\tau: F \Rightarrow G$ is a family of $\left\{\tau_{a}: F a \rightarrow G a\right\}_{a \in \mathrm{A}_{0}}$ of morphisms in $\mathrm{B}$, one for each object $a$ of $\mathrm{A}$, such that, for any $f \in \operatorname{Hom}_{\mathrm{A}}\left(a, a^{\prime}\right)$, the following diagram commutes:

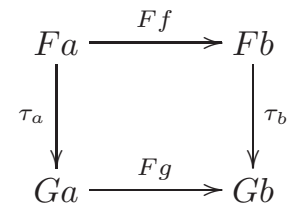

If each $\tau_{a}$ is an isomorphism, we say that $\tau$ is a natural isomorphism (an older term is natural equivalence).

Definition A.21 (Equivalence of categories). An equivalence of categories consists of a pair of functors

$$
F: \mathrm{A} \rightarrow \mathrm{B}, \quad E: \mathrm{B} \rightarrow \mathrm{A}
$$

and a pair of natural isomorphisms

$$
\alpha: 1_{\mathrm{A}} \Rightarrow E \circ F \quad \beta: 1_{\mathrm{B}} \Rightarrow F \circ E .
$$

In this situation the functor $F$ is called the pseudo-inverse or the homotopy inverse of $E$. The categories $A$ and $\mathrm{B}$ are then said to be equivalent categories.

Proposition A.22. A functor $F: A \rightarrow B$ is (part of) an equivalence of categories if and only if it is fully faithful and essentially surjective.

Proof. See [2, Proposition 7.25]

Example A.23. The categories Mat of matrices and FinVect of finite dimensional vector spaces are equivalent categories since the functor $\iota:$ Mat $\rightarrow$ FinVect (q.v. Example A.15) is fully faithful and essentially surjective. Note that the functor $\iota$ is not surjective on objects.

\section{Appendix B. Densities}

In this section we borrow from a manuscript by Guillemin and Sternberg [8].

B.1. Densities on a vector space. Consider an $n$-dimensional real vector space $V$. Recall that a basis $\left\{v_{1}, \ldots, v_{n}\right\}$ defines a linear isomorphism $\vee: \mathbb{R}^{n} \rightarrow V$ by $\vee\left(x_{1}, \ldots, x_{n}\right)=\sum x_{i} v_{i}$. Conversely any linear isomorphism $\vee: \mathbb{R}^{n} \rightarrow V$ defines a basis $\left\{v_{1}, \ldots, v_{n}\right\}$ of $V$ by setting the $i^{\text {th }}$ basis vector $v_{i}$ to be the image $\mathrm{v}\left(e_{i}\right)$ of the standard $i^{\text {th }}$ basis vector $e_{i}$ of $\mathbb{R}^{n}$ under the isomorphism $v$. From now on we will not distinguish between a basis of $V$ and a linear isomorphism from $\mathbb{R}^{n}$ to $V$.

Definition B.1 (Frame). We denote the space of bases of an $n$ dimensional real vector space $V$ by $\mathcal{F} r(V)$ and refer to it as the space of frames of $V$.

Note that there is a natural right action $\bullet$ of the Lie group $G L(n, \mathbb{R}) \equiv G L\left(\mathbb{R}^{n}\right)$ on the space of frames $\mathcal{F} r(V)$ of an $n$-dimensional vector space $V$ by composition on the right:

$$
\vee \bullet A:=\mathrm{v} \circ A
$$

for all isomorphisms $\vee: \mathbb{R}^{n} \rightarrow V$ and all $A \in G L(n, \mathbb{R})$. Moreover this action is free and transitive: given $\mathrm{v}, \mathrm{v}^{\prime} \in \mathcal{F} r(V)$ and $A \in G L(n, \mathbb{R})$

$$
\mathrm{v} \bullet A=\mathrm{v} \quad \text { if and only if } A=\left(\mathrm{v}^{-1} \circ \mathrm{v}^{\prime}\right) .
$$

Remark B.2. A space $X$ with a free and transitive action of a group $G$ is called a $G$ torsor.

With these preliminaries out of the way we are ready to define $\alpha$-densities on a vector space.

Definition B.3 ( $\alpha$-density). Let $\alpha$ denote a complex number. An $\alpha$-density (also called a density of order $\alpha$ ) on an $n$-dimensional real vector space $V$ is a map

$$
\tau: \mathcal{F} r(V) \rightarrow \mathbb{C} \quad \text { with } \quad \tau(\mathrm{v} \bullet A)=\tau(\mathrm{v})|\operatorname{det}(A)|^{\alpha}
$$

for all $\vee \in \mathcal{F} r(V)$ and all $A \in G L(n, \mathbb{R})$.

Notation B.4. Since $\alpha$-densities on a fixed vector space $V$ are complex valued functions, they form a complex vector space. We denote it by $|V|^{\alpha}$. In other words

$$
|V|^{\alpha}:=\left\{\tau:\left.\mathcal{F} r(V) \rightarrow \mathbb{C}|\tau(\mathrm{v} \bullet A)=\tau(\mathrm{v})| \operatorname{det}(A)\right|^{\alpha} \text { for all } \mathrm{v} \in \mathcal{F} r(V), A \in G L(n, \mathbb{R})\right\} .
$$


Remark B.5. Alternatively one may view the space of frames $\mathcal{F} r(V)$ as a principal $G L(n, \mathbb{R})$ bundle over a point. An $\alpha$-density is then a section of the associated bundle $(\mathcal{F} r(V) \times \mathbb{C}) / G L(n, \mathbb{R})$, where $G L(n, \mathbb{R})$ acts on $\mathbb{C}$ by the character $A \mapsto|\operatorname{det} A|^{\alpha}$. Since $(\mathcal{F} r(V) \times \mathbb{C}) / G L(n, \mathbb{R})$ is a complex line bundle over a point, it is a one dimensional complex vector space. Hence the space of sections of this bundle, i.e., the space of densities $|V|^{\alpha}$, has complex dimension 1. In particular we have proved:

Lemma B.6. The space $|V|^{\alpha}$ of $\alpha$-densities on a vector space $V$ is a complex 1-dimensional vector space.

Remark B.7. Any nonzero $n$-form $\omega \in \Lambda^{n}\left(V^{*}\right)$ on an $n$-dimensional real vector space $V$ defines an $\alpha$-density $|\omega|^{\alpha}$ by the formula

$$
|\omega|^{\alpha}\left(v_{1}, \ldots, v_{n}\right):=\left|\omega\left(v_{1}, \ldots, v_{n}\right)\right|^{\alpha}
$$

for all frames $\left\{v_{1}, \ldots, v_{n}\right\} \in \mathcal{F} r(V)$.

Conversely, since the space $|V|^{\alpha}$ of $\alpha$ densities is 1-dimensional, any density $\tau \in|V|^{\alpha}$ is of the form $\tau=c|\omega|^{\alpha}$ for some nonzero $n$-form $\omega \in \Lambda^{n}\left(V^{*}\right)$ and a constant $c \in \mathbb{C}$.

Remark B.8. Densities pull back under linear isomorphisms. If $T: W \rightarrow V$ is an isomorphism of $n$-dimensional vector spaces and $\tau \in|V|^{\alpha}$ is a density then its pullback $T^{*} \tau: \mathcal{F} r(W) \rightarrow \mathbb{C}$ is defined by

$$
T^{*} \tau\left(w_{1}, \ldots w_{n}\right)=\tau\left(T w_{1}, \ldots T w_{n}\right)
$$

for any frame $\mathbf{w}=\left\{w_{1}, \ldots, w_{n}\right\} \in \mathcal{F} r(W)$. Note that since $T$ is an isomorphism the tuple $\left(T w_{1}, \ldots T w_{n}\right)$ is a frame of $V$, so the definition of pullback does make sense.

Remark B.9. Densities can be multiplied: if $\rho \in|V|^{\alpha}$ and $\tau \in|V|^{\beta}$ are two densities of order $\alpha$ and $\beta$ respectively then their product defined by

$$
(\rho \cdot \tau)(\mathrm{v})=\rho(\mathrm{v}) \tau(\mathrm{v})
$$

is easily seen to be a density of order $\alpha+\beta$. Since the multiplication map $(\rho, \tau) \mapsto \rho \cdot \tau$ is $\mathbb{C}$ bilinear, we get a $\mathbb{C}$ linear map

$$
|V|^{\alpha} \otimes|V|^{\beta} \rightarrow|V|^{\alpha+\beta} .
$$

Since the map is nonzero, it is an isomorphism of vector spaces by dimension count. In particular we have a canonical isomorphism

$$
|V|^{1 / 2} \otimes|V|^{1 / 2} \stackrel{\sim}{\rightarrow}|V|^{1} .
$$

Remark B.10. It makes sense to take a complex conjugate of a density. If $\rho \in|V|^{\alpha}$ is an $\alpha$-density then $\bar{\rho}: \mathcal{F} r(V) \rightarrow$ $\mathbb{C}$ defined by

$$
\bar{\rho}(\mathrm{v}):=\overline{\rho(\mathrm{v})}
$$

for all $\vee \in \mathcal{F} r(V)$ is easily seen to be an $\bar{\alpha}$-density.

Therefore we can define on the space $|V|^{1 / 2}$ of half-densities a $|V|^{1}$-valued Hermitian inner product by

$$
(\mu, \tau):=\bar{\mu} \tau .
$$

B.2. Densities on manifolds. Recall that for any real vector bundle $E \rightarrow M$ of rank $k$ over a manifold $M$ we have the principal $G L(k, \mathbb{R})$ bundle $\mathcal{F} r(E) \rightarrow M$, the so-called frame bundle of $E \rightarrow M$. A typical fiber $\mathcal{F} r(E)_{q}$ of this bundle above a point $q$ of $M$ consists of the frames of the fiber $E_{q}$ of the bundle $E$ :

$$
\mathcal{F} r(E)_{q}:=\mathcal{F} r\left(E_{q}\right) .
$$

Recall also that we can think of $\mathcal{F} r(E)$ as an open subset of the vector bundle $\operatorname{Hom}\left(M \times \mathbb{R}^{k}, E\right) \rightarrow M ; \mathcal{F} r(E)$ consists of isomorphisms. There is a natural right $G L(k, \mathbb{R})$ action of $\mathcal{F} r(E)$ making it a principal $G L(k, \mathbb{R})$ bundle.

Next recall that given a principal $G$-bundle $G \rightarrow P \rightarrow M$ over a manifold $M$ and a (complex) representation $\rho: G \rightarrow G L(W)$, we can build out of this data a complex vector bundle $P \times{ }^{\rho} W \rightarrow M$ over $M$. This bundle is the quotient of the trivial bundle $P \times W$ by a free and proper left action of $G$ :

$$
P \times{ }^{\rho} W:=(P \times W) / G, \quad g \cdot(p, w):=\left(p g^{-1}, \rho(g) w\right) .
$$

It will be useful to recall that the space of sections of the associated bundle $\Gamma\left(P \times^{\rho} W\right)$ is isomorphic to the space of equivariant $W$-valued functions on $P$ :

$$
\Gamma\left(P \times{ }^{\rho} W\right) \simeq\left\{\varphi: P \rightarrow W \mid \varphi(p \cdot g)=\rho(g)^{-1} \varphi(p)\right\} .
$$


Definition B.11 (densities on a manifold). We define the complex line bundle $|T M|^{\alpha} \rightarrow M$ of $\alpha$-densities on a manifold $M$ to be the associated bundle

$$
|T M|^{\alpha}:=\mathcal{F} r(T M) \times^{|\operatorname{det}|^{-\alpha}} \mathbb{C},
$$

where the representation $|\operatorname{det}|^{-\alpha}: G L(k, \mathbb{R}) \rightarrow G L(\mathbb{C})$ is defined by

$$
|\operatorname{det}|^{-\alpha}(A) z:=|\operatorname{det} A|^{-\alpha} z \quad \text { for all } \quad A \in G L(k, \mathbb{R}), z \in \mathbb{C} .
$$

We refer to the sections of the bundle $|T M|^{\alpha} \rightarrow M$ as $\alpha$-densities on the manifold $M$. We denote the space of $\alpha$-densities on a manifold $M$ by $|M|^{\alpha}$ :

$$
|M|^{\alpha}:=\Gamma\left(\mathcal{F} r(T M) \times^{|\operatorname{det}|^{-\alpha}} \mathbb{C}\right) .
$$

Remark B.12. We may and will identify the space of $\alpha$-densities on a manifold $M$ with the space of equivariant complex functions on its frame bundle:

$$
|M|^{\alpha} \simeq\left\{\tau:\left.\mathcal{F} r(T M) \rightarrow \mathbb{C}|\tau(\mathrm{v} \bullet A)=| \operatorname{det} A\right|^{\alpha} \tau(\mathrm{v}) \text { for all } A \in G L(m, \mathbb{R}), \mathrm{v} \in \mathcal{F} r(T M)\right\} .
$$

Remark B.13. Note that by design the fiber of the bundle $|T M|^{\alpha} \rightarrow M$ at a point $q$ is the 1-dimensional complex vector space $\left|T_{q} M\right|^{\alpha}$ of $\alpha$-densities on the tangent space $T_{q} M$.

Remark B.14. By construction the transition maps for the complex line bundle $|T M|^{\alpha} \rightarrow M$ take their values in positive real numbers. It follows that $|T M|^{\alpha} \rightarrow M$ is a trivial bundle but not canonically. In particular its space of sections $|M|^{\alpha}$ is a rank $1 C^{\infty}(M, \mathbb{C})$ module. Since the space of sections of the tensor product is isomorphic to the tensor product of sections (as $C^{\infty}(M)$ modules), it follows that for any complex line bundle $\mathbb{L} \rightarrow M$, a section of $\mathbb{L} \otimes|T M|^{\alpha}$ is of the form $s \otimes \tau$ for some section $s \in \Gamma(\mathbb{L})$ of $\mathbb{L} \rightarrow M$ and some $\alpha$-density $\tau$.

Remark B.15. Since densities on a vector space pull back under a linear isomorphism, densities on a manifold pull back under local diffeomorphisms: If $F: N \rightarrow M$ is a local diffeomorphism between two manifolds and $\tau: \mathcal{F} r(T M) \rightarrow \mathbb{C}$ an $\alpha$-density on $M$, the pullback $F^{*} \tau \in|N|^{\alpha}$ is defined as follows: for any point $q \in N$ and any frame $\left(v_{1}, \ldots, v_{n}\right)$ of $T_{q} N$

$$
\left(F^{*} \tau\right)_{q}\left(v_{1}, \ldots, v_{n}\right):=\tau_{F(q)}\left(d F_{q} v_{1}, \ldots, d F_{q} v_{n}\right) .
$$

Remark B.16. For any open subset $U$ of $\mathbb{R}^{m}$ we have the canonical $\alpha$-density $\left|d x_{1} \wedge \cdots d x_{m}\right|^{\alpha}$ (q.v. Remark B.7). Here, of course, $x_{1}, \ldots, x_{m}$ are the Cartesian coordinates on $\mathbb{R}^{m}$. This density defines an isomorphism $U \times \mathbb{C} \rightarrow$ $|T U|^{\alpha}$. Therefore, for any $\alpha$-density $\tau$ on $U$ there exists a unique function $f_{\tau} \in C^{\infty}(U)$ with

$$
\tau=f_{\tau}\left|d x_{1} \wedge \cdots d x_{n}\right|^{\alpha} .
$$

Indeed,

$$
f_{\tau}=\tau\left(\frac{\partial}{\partial x_{1}}, \ldots, \frac{\partial}{\partial x_{m}}\right) \text {. }
$$

Putting the preceding remarks together we note that for a diffeomorphism $F: U \rightarrow V$ of open subsets of $\mathbb{R}^{m}$ and an $\alpha$-density $f(y)\left|d y_{1} \wedge \ldots \wedge d y_{m}\right|^{\alpha}$ on $V$ we have

$$
F^{*}\left(f(y)\left|d y_{1} \wedge \ldots \wedge d y_{m}\right|^{\alpha}\right)=f(F(x))\left|\operatorname{det} d F_{x}\right|^{\alpha}\left|d x_{1} \wedge \cdots \wedge d x_{n}\right|^{\alpha} .
$$

Formula (B.1) is a reason why 1-densities can be integrated over manifolds. The story parallels the familiar story of integration of top degree forms on oriented manifolds. The 1-densities have the advantage that the manifold doesn't have to be oriented (or even be orientable) for their integrals to make sense.

The story proceeds as follows. If $U \subset \mathbb{R}^{n}$ is an open set and $\tau \in|U|^{1}$ a 1-density, then, as remarked above,

$$
\tau=f_{\tau}\left|d x_{1} \wedge \ldots \wedge d x_{n}\right|
$$

for a unique complex valued smooth function $f_{\tau}$ on $U$. One defines the integral $\int_{U} \tau$ by

$$
\int_{U} \tau:=\int_{U} f_{\tau}
$$

Or, if you prefer,

$$
\int_{U} f\left|d x_{1} \wedge \ldots \wedge d x_{n}\right|:=\int_{U} f d x_{1} \ldots d x_{n}
$$

for any smooth integrable function $f$ on the set $U$. This is a complex valued integral.

If $M$ is a manifold of dimension $m, \phi: U \rightarrow \mathbb{R}^{m}$ a coordinate chart and $\tau$ is a 1-density on $M$ with support in $U$ one defines

$$
\int_{M} \tau:=\int_{\phi(U)}\left(\phi^{-1}\right)^{*} \tau
$$


If $\psi: U \rightarrow \mathbb{R}^{m}$ is another coordinate chart, then

$$
\left(\psi^{-1}\right)^{*} \tau=f(y)\left|d y_{1} \wedge \cdots \wedge d y_{m}\right|
$$

for some $f \in C^{\infty}(\psi(U))$. Consider the diffeomorphism $F=\psi \circ \phi^{-1}: \phi(U) \rightarrow \psi(U)$. We have

$$
\left(\phi^{-1}\right)^{*} \tau=\left(\psi^{-1} \circ \psi \phi^{-1}\right)^{*} \tau=F^{*}\left(\psi^{-1}\right)^{*} \tau .
$$

By (B.1)

$$
\left(\phi^{-1}\right)^{*} \tau=f(F(x))\left|\operatorname{det} D F_{x}\right|\left|d x_{1} \wedge \cdots \wedge d x_{m}\right| .
$$

Therefore

$$
\int_{\phi(U)}\left(\phi^{-1}\right)^{*} \tau=\int_{\phi(U)} f(F(x))\left|\operatorname{det} D F_{x}\right| d x_{1} \cdots d x_{m}=\int_{F(\phi(U))=\psi(U)} f(y) d y_{1} \cdots d y_{m}=\int_{\psi(U)}\left(\psi^{-1}\right)^{*} \tau, .
$$

where the second equality holds by the change of variables formula for functions on regions of $\mathbb{R}^{m}$. Therefore integrals of 1-densities supported in coordinate charts are well-defined.

Next one makes sense of integrability of non-negative densities. A 1-density $\tau$ on a manifold $M$ is non-negative if its value at any frame $\left\{v_{1}, \ldots v_{m}\right\} \subset T_{q} M$ is a non-negative real number. It is not hard to see that "non-negativity" is well-defined. Now choose a locally finite cover $\left\{U_{\alpha}\right\}$ of $M$ by coordinate charts and choose a partition of unity $\left\{\rho_{\alpha}\right\}$ subordinate to the cover. We say that a non-negative 1-density is integrable if the sum

$$
\sum_{\alpha} \int_{M} \rho_{\alpha} \tau=\sum_{\alpha} \int_{U_{\alpha}} \rho_{\alpha} \tau
$$

converges. We then define $\int_{M} \tau$ to be the sum:

$$
\int_{M} \tau:=\sum_{\alpha} \int_{U_{\alpha}} \rho_{\alpha} \tau
$$

The rest of the definition of integration of 1-densities proceeds just like for functions. A given real-valued 1-density $\tau$ can be written as a difference of two (continuous) non-negative 1-densities $\tau_{+}$and $\tau_{-}$:

$$
\tau=\tau_{+}-\tau_{-} .
$$

We call $\tau$ integrable if $\int_{M} \tau_{+}$and $\int_{M} \tau_{-}$are finite. We then set $\int_{M} \tau=\int_{M} \tau_{+}-\int_{M} \tau_{-}$. Finally we define a complex valued 1-density $\tau$ integrable if its real and imaginary parts are integrable; we define its integral to be the sum

$$
\int_{M} \tau=\int_{M} \operatorname{Re}(\tau)+\sqrt{-1} \int_{M} \operatorname{Im}(\tau)
$$

B.3. The "Intrinsic" Hilbert space. Suppose $(\mathbb{L} \rightarrow M,\langle\cdot, \cdot\rangle)$ is a Hermitian line bundle. Then given a 1-density $\tau$ on $M$ we can define a Hermitian pairing pairing of sections of $\mathbb{L}$ by

$$
\left\langle\left\langle s, s^{\prime}\right\rangle\right\rangle:=\int_{M}\left\langle s, s^{\prime}\right\rangle \tau
$$

There is an associated Hilbert space of "square integrable" sections, which, of course, depends on the choice of our density $\tau$. There is also a more intrinsic pairing of sections of a slightly different bundle. Consider the tensor product of complex line bundles $\mathbb{L} \otimes|M|^{1 / 2} \rightarrow M$. By Remark B.14 a section of $\mathbb{L} \otimes|M|^{1 / 2} \rightarrow M$ is of the form $s \otimes \mu$, where $s \in \Gamma(\mathbb{L})$ and $\mu$ is a $\frac{1}{2}$-density. Now given two sections $s_{1} \otimes \mu_{1}$ and $s_{2} \otimes \mu_{2}$ we can pair them to get a 1-density $\left\langle s_{1}, s_{2}\right\rangle \bar{\mu}_{1} \mu_{2}$ (q.v. Remark B.10). Hence the Hermitian inner product

$$
\Gamma\left(\mathbb{L} \otimes|M|^{1 / 2}\right) \times \Gamma\left(\mathbb{L} \otimes|M|^{1 / 2}\right) \rightarrow \mathbb{R}, \quad\left\langle\left\langle s_{1} \otimes \mu_{1}, s_{2} \otimes \mu_{2}\right\rangle\right\rangle:=\int_{M}\left\langle s_{1}, s_{2}\right\rangle \bar{\mu}_{1} \mu_{2}
$$

makes sense (whenever the integral converges). It is easy to see that the integral above does converge for all sections in the space

$$
L^{2}\left(\mathbb{L} \otimes|M|^{1 / 2}, M\right) \cap \Gamma\left(\mathbb{L} \otimes|M|^{1 / 2}\right):=\left\{\left.s \otimes \mu \in \Gamma\left(\mathbb{L} \otimes|M|^{1 / 2}\right)\left|\int_{M}\langle s, s\rangle\right| \mu\right|^{2}<\infty\right\} .
$$

The completion of the space with respect to the Hermitian inner product gives us the "intrinsic Hilbert space of square-integrable sections" $L^{2}\left(\mathbb{L} \otimes|M|^{1 / 2}, M\right)$. 


\section{REFERENCES}

[1] S.T. Ali and M. Englis, Quantization methods: a guide for physicists and analysts, Rev. Math. Phys. 17(2005), no. 4, 391-490.

[2] Steve Awodey. Category theory, volume 49 of Oxford Logic Guides. The Clarendon Press Oxford University Press, New York, 2006.

[3] S. Bates and A. Weinstein, Lectures on the geometry of quantization, Berkeley Mathematics Lecture Notes, 8. American Mathematical Society, Providence, RI; Berkeley Center for Pure and Applied Mathematics, Berkeley, CA, 1997. vi+137 pp. ISBN: 0-8218-0798-6

[4] J.J. Duistermaat, On global action-angle coordinates. Comm. Pure Appl. Math. 33 (1980), no. 6, 687-706.

[5] A. Echeverria-Enriquez, M.C. Munoz-Lecanda, N. Roman-Roy and C. Victoria-Monge, Mathematical foundations of geometric quantization, Extracta Math. 13(1998), 135-238.

[6] M. Gotay, A class of nonpolarizable symplectic manifolds, Monatsh. Math., 103 (1987) 27-30; doi 10.1007/BF01302708.

[7] V. Guillemin and S. Sternberg, Geometric Asymptotics, Mathematical Surveys, No. 14. American Mathematical Society, Providence, R.I., 1977. xviii+474 pp.

[8] V. Guillemin and S. Sternberg, Semi-classical analysis, http://www-math.mit.edu/ vwg/semiclassGuilleminSternberg.pdf, accessed on 1/09/2012.

[9] M.J. Hopkins and I.M. Singer, Quadratic functions in geometry, topology, and M-theory, J. Differential Geom., 70 (2005),329452.

[10] V. Guillemin, V. Ginzburg and Y. Karshon, Moment maps, cobordisms, and Hamiltonian group actions, Appendix J by Maxim Braverman. Mathematical Surveys and Monographs, 98. American Mathematical Society, Providence, RI, 2002. viii+350 pp. ISBN: 0-8218-0502-9

[11] E. Lerman and A. Malkin, Differential characters as stacks and prequantization, J. Gokova Geom. Topol. GGT 2 (2008), $14-39$.

[12] J. Sniatycki, Geometric quantization and quantum mechanics, Applied Mathematical Sciences, 30. Springer-Verlag, New YorkBerlin, 1980. ix+230 pp. ISBN: 0-387-90469-7

[13] D.J. Simms and N.M.J. Woodhouse, Lectures in geometric quantization, Lecture Notes in Physics, 53. Springer-Verlag, BerlinNew York, 1976. iii+166 pp. ISBN: 3-540-07860-6

[14] J.-M. Souriau,Structure of dynamical systems. A symplectic view of physics. Progress in Mathematics, 149. Birkhäuser Boston, Inc., Boston, MA, 1997. xxxiv+406 pp. ISBN: 0-8176-3695-1 .

[15] N.R. Wallach, Symplectic geometry and Fourier analysis, with an appendix on quantum mechanics by Robert Hermann. Lie Groups: History, Frontiers and Applications, Vol. V. Math Sci Press, Brookline, Mass., 1977. xvii+436 pp.

[16] N.M.J. Woodhouse, Geometric quantization, Second edition. Oxford Mathematical Monographs. Oxford Science Publications. The Clarendon Press, Oxford University Press, New York, 1992. xii+307 pp. ISBN: 0-19-853673-9 